\title{
UNIFORM TRANSFORMATION GROUPS ON LOWER DIMENSIONAL SPACES
}

\author{
YUAN TIAN
}

\begin{abstract}
.
In this paper we study transformation groups which are not assumed (locally) compact. We study transformation groups with uniformly continuous group multiplication and generalize some results for (locally) compact groups. The main theorem is that if a uniform group $G$ acts openly as a transformation group on Euclidean 2-space $R^{2}$, then any arcwise connected and effective subgroup $G_{0}$ of $G$, which fixes a point of $R^{2}$, is commutative. Moreover, unless $G_{0}=\{e\}$, there is an open, connected and dense subset $O$ of $R^{2}$ such that, every orbit $G_{0} x$ of $x \in O$ is either a Jordan curve or is homeomorphic with the real line $R$ (when it is homeomorphic with the real line, it is unbounded); $G_{0}$ has at most two fixed points in $R^{2}$ and in case $G_{0}$ has exactly two fixed points, we have proved that every orbit $G_{0} x$ of $x \in R^{2}$ other than the two fixed points is either a Jordan curve, or is homeomorphic with the real line $R$ (then it is unbounded).
\end{abstract}

\section{Introduction.}

The study of transformation groups originated in the use of groups in geometry (in the work of Lie on continuous groups, and in the work of Poincare on dynamical systems). Three standard works to the theory are(1) Montgomery and Zippin, Topological Transformation Groups 1955 [1], (2) Borel et al., Seminar on Transformation Groups, 1960 [2], and (3) Conner and Floyd, Differential Periodic maps, 1964 [3].

A transformation group of a space is a pair $(G, M)$ where $G$ is a topological group, $M$ is a Hausdorff space and where further to each element $g \in G$ there is given a homeomorphism $x \rightarrow g(x)$ of $M$ onto itself satisfying

1) $g(x)$ is simultaneously continuous in $g$ and $x$;

2) $g_{1}\left(g_{2}(x)\right)=\left(g_{1} g_{2}\right)(x)$.

There are many different levels of generality on which questions may be raised and on all of these levels there are many unsolved problems. In case when $G$ is a (locally) compact topological group and $M$ is a manifold, the theory has been developed considerably. 
In this paper we study transformation groups which are not (locally) compact, but which act on a low-dimensional space. We do not make any differentiability assumptions on the transformations.

In Enflo [4], the (local) uniform continuity of the group multiplication is an essential assumption and topological groups with locally uniformly continuous group multiplication are studied. Since a (locally) compact group is always a (locally) uniform group, the concept "(locally) uniform group" in some sense generalizes the concept "(locally) compact group". It is natural to investigate (locally) uniform group by generalizing some results for (locally) compact groups.

We have the following results in [1] for compact transformation groups:

"Let $G$ be a compact connected group which acts on Euclidean three-space $R^{3}$ effectively. Then $G$ is either the circle group or the proper orthogonal group in three variables".

The circle group is commutative group. The proper orthogonal group in three variables is not commutative, but any subgroup which fixes a point is commutative.

Similar results also hold for Euclidean 2-space $R^{2}$ and Euclidean 1-space $R^{1}$.

Consider the corresponding problem for uniform groups. The main theorem (Theorem 3.4.6) in this paper says that, with somewhat stronger assumptions, we have similar result under the more general assumption "uniform group" instead of "compact group".

In Enflo [4] the following theorem was proved:

"If a locally generated, uniform group acts effectively as a transformation group on the real line, then it is commutative".

The concept " $G$ is locally generated" is a generalization of the concept " $G$ is connected" since a connected group is a locally generated group (see Proposition 1.2.11 in this paper).

We carry over Enflo's theorem to the case when $G$ is a uniform transformation group on the unit circle $C$ in Chapter 2 of this paper. We prove

(1) If a connected, uniform topological group $G$ acts effectively as a transformation group on the unit circle $C=\{z,|z|=1\}$, then $G$ is commutative. (See Theorem 2.2.3)

Next we study uniform transformation groups on Euclidean 2-space $R^{2}$. Our main theorem in this paper is

(2) Let $G$ be a uniform topological group that acts openly as a transformation group on $R^{2}$. For a point $x_{0} \in R^{2}$, let $G_{0}$ be the subgroup of $G$, which consists of all 
the transformations that fix $x_{0}$. We assume that $G_{0}$ is arcwise connected. If $G_{0}$ acts effectively on $R^{2}$, then $G_{0}$ is a commutative subgroup of $G$. (See Theorem 3.4.6)

The proof of this theorem is based on the knowledge about the orbits $G_{0} x$ of $x \in R^{2}$ under $G_{0}$. It turns out that "almost" every orbit $G_{0} x$ of $x \in R^{2}$ is as good as either a Jordan curve (which is homeomorphic with the unit circle $C$ ) or it is homeomorphic with the real line $R$, if $G_{0}$ does not leave every point of $R^{2}$ fixed. In fact, we show in Chapter 3 the following

(3) If $G_{0}$ does not leave every point of $R^{2}$ fixed, then there is an open, connected and dense subset $O$ of $R^{2}$, such that, every orbit $G_{0} x$ with $x \in O$ is either a Jordan curve or homeomorphic with the real line $R$ (then it is unbounded) (See Theorem 3.4.3).

Besides, we have good knowledge about the fixed point set of $G_{0}$ in $R^{2}$ as follows:

(4) If $G_{0}$ does not fix every point of $R^{2}$, then it fixes at most two points of $R^{2}$ (See Theorem 3.4.4).

(5) In case $G_{0}$ fixes exactly two points of $R^{2}$, every orbit $G_{0} x$ of $x \in R^{2}$ other than the two fixed points is either a Jordan curve or it is homeomorphic with the real line $R$ (then it is unbounded). (See Corollary 3.4.5)

The results (3), (4) and (5) are true without assuming that $G_{0}$ acts effectively on $R^{2}$. Using these results, we are able to conclude that $G_{0}$, as a transformation group on $R^{2}$, acts commutatively at least on an open, connected and dense subset of $R^{2}$. This easily implies that $G_{0}$ acts commutatively on $R^{2}$. Moreover, if $G_{0}$ acts effectively on $R^{2}$, it is easy to show that $G_{0}$ is a commutative subgroup of $G$.

The results about orbits $G_{0} x$ of $x \in R^{2}$ under $G_{0}$ in this paper ((3), (4) and (5)) have not only been tools for showing that $G_{0}$ is a commutative subgroup of $G$. They are as well interesting in themselves. Actually, they can be considered as generalizations of the following results in [1] for compact transformation groups:

"If a compact group $G$ acts on a locally Euclidean n-dimensional space E then any $(n-1)$-dimensional orbit is locally connected."

"Let $G$ be a compact connected group which acts on Euclidean $n$-space $E$ with at least one $(n-1)$-dimensional orbit. Then all orbits except one are $(n-1)$-dimensional".

This paper is organized as follows:

In chapter 1 we give definitions and some general properties and results which will be useful for the proofs of the theorems in this paper. In chapter 2 we prove result (1) and in chapter 3 we prove results (2), (3), (4) and (5). 


\section{Definitions and general results.}

\subsection{DEFINITIONS.}

1. Transformation group of a space. A transformation group of a space is defined as a pair $(G, M)$ where $G$ is a topological group, $M$ is a topological Hausdorff space and where further to each element $g \in G$ there is given a homeomorphism $x \rightarrow g(x)$ of $M$ onto itself satisfying

1) $g(x)$ is simultaneously continuous in $g$ and $x$;

2) $g_{1}\left(g_{2}(x)\right)=\left(g_{1} g_{2}\right)(x)$.

From 2) and from the fact that each $g$ is one-one on $M$, it follows that for every $x \in M$

3) $e(x)=x ; e$ is the identity in $G$.

If $e$ is the only element in $G$ which leaves all of $M$ fixed, i.e. if $e$ is the only element satisfying 3 ) for all $x$, then $G$ is called effective.

A transformation group $G$ is called transitive on $M$ if for every $x, y \in M$ there is at least one $g \in G$, such that $g(x)=y$.

We say that $G$ acts openly as a transformation on $M$ if $g \rightarrow g(x)$ is an open mapping $G \rightarrow M$, for every fixed $x \in M$.

We say that $G$ acts commutatively on $M$, if for every pair of $g, f \in G$ and for every $x \in M$, we have $g f(x)=f g(x)$.

2. Uniform group. For the definition of uniform space the reader is referred to Kelly [5]. We recall that if $G$ is a topological group, then the left uniformity for $G$ is the uniformity which has as a base the family of sets $\left\{(x, y) \mid x^{-1} y \in U\right\}$ where $U$ runs through the neighborhoods of $e$ in $G$. The right uniformity is defined in the same way but with $x y^{-1}$ instead of $x^{-1} y$.

We say that a topological group $G$ is a uniform group if there is a uniform structure $\mathscr{U}$ for $G$ such that $(x, y) \rightarrow x y$ is uniformly continuous with respect to $\mathscr{U}$.

In Enflo [4], it is proved that $G$ is a uniform group if and only if the left and the right uniformity for $G$ coincide. Then the uniformity of $G$ coincides with the left and right uniformities and $(x, y) \rightarrow x y$ is uniformly continuous as a function $G \times G \rightarrow G, x \rightarrow x^{-1}$ is uniformly continuous as a function $G \rightarrow G$. Besides, there is the following useful lemma in [4]:

" $G$ is a uniform group if and only if for every neighborhood $U$ of $e$ there is a neighborhood $V$ of $e$ such that for all $x \in G, x^{-1} V x \subset U$."

In this paper, we use this necessary and sufficient condition as the definition of uniform groups.

3. Orbit. Let $(G, M)$ be a transformation group and $H$ a subgroup of $G$. Then $(H, M)$ is also a transformation group. For each $x \in M$, the set 


$$
H(x)=\{h(x), h \in H\} \subset M
$$

is called the orbit of $x$ under $H$.

4. Locally generated groups. A topological group $G$ is called locally generated, if for every neighborhood $V$ of $e$ the smallest subgroup of $G$ which contains $V$ is $G$.

5. Pathwise (Arcwise) connected spaces. A topological space $X$ is called pathwise (arcwise) connected if for any two points $x$ and $y$ in $X$, there is a continuous function (homeomorphism) $f:[0,1] \rightarrow X$ such that $f(0)=x, f(1)=y$. The function $f$ (as well as its range) is called a path (arc) from $x$ to $y$.

6. $n$-Manifold. An $n$-dimensional manifold is a Hausdorff space on which there exists an open covering by sets homeomorphic with open sets in $R^{n}$.

By this definition, we see that a Hausdorff space $X$ is a 1-manifold, if for every point $x$ of $X$, there is an open neighborhood of $x$ homeomorphic with the open unit interval $(0,1)$. Then we also use the expression that $X$ at $x$ is locally an arc.

7. Dimension. The empty set and only the empty set has dimension -1 . A space $X$ has dimension $\leqq n(n \geqq 0)$ at a point $p$ if $p$ has arbitrarily small neighborhoods whose boundaries have dimension $\leqq n-1$. $X$ has dimension $\leqq n, \operatorname{dim} X \leqq n$, if $X$ has dimension $\leqq n$ at each of its points. $X$ has dimension $n$ at a point $p$ if it is true that $X$ has dimension $\leqq n$ at $p$ and it is false that $X$ has dimension $\leqq n-1$ at $p$. $X$ has dimension $n$ if $\operatorname{dim} X \leqq n$ is true and $\operatorname{dim} X \leqq n-1$ is false.

By this definition, Euclidean $n$-space has dimension $n[6]$.

8. Accessibility by arc and by set from a point set. A point $a$ is called arcwise accessible from a point set $B$ if $b \in B$ implies the existence of an $\operatorname{arc} T$ with end points $a$ and $b$ such that $T-a \subset B$. If $A$ is a point set every point of which is arcwise accessible from some point set $B$, then we call $A$ arcwise accessible from $B$. That a point $p$ is accessible from $B$ by closed and connected sets means that for each $q \in B$, there exists a closed and connected set $K$ which contains $p$ and $q$ such that $K-p \subset B$.

9. Domain. A domain is an open and connected subset of a topological space.

\subsection{SOME GENERAL PROPERTIES AND RESULTS.}

Proposition 1.2.1. Let $(G, M)$ be a transformation group and $H$ a subgroup of $G$. For each $x \in M$, consider the orbit $H(x)=\{h(x), h \in H\}$. Then we have

a) $H(x)$ is connected, if $H$ is a connected subgroup of $G$.

b) $(H, H(x))$ is also a transformation group and $H$ acts transitively on $H(x)$. 
c) We have either $H\left(x_{1}\right) \equiv H\left(x_{2}\right)$ or $H\left(x_{1}\right) \cap H\left(x_{2}\right)=\emptyset$, for every two $x_{1}, x_{2} \in M$.

Proof. The proofs are trivial.

Proposition 1.2.2. Let $(G, M)$ be a transformation group. Then for every open set $O$ in $M$ and for every compact set $K \subset O \subset M$, there exists a neighborhood $U$ of $e$ in $G$, such that $u(x) \in O$, for all $u \in U, x \in K$.

Proof. Since $(G, M)$ is a transformation group, for every $x \in K$ (then $O$ is an open neighborhood of $x$ ) there exists a neighborhood $O_{x}$ of $x$ in $M$ and a neighborhood $U_{x}$ of $e$ in $G$, such that $u(y) \in O$, for all $u \in U_{x}, y \in O_{x}$. $K$ is compact, so there exist finitely many $x$ 's in $K$, say $x_{1}, x_{2}, \ldots, x_{n}$, such that $\left\{O_{x_{i}}, i=1,2, \ldots, n\right\}$ is a finite open cover of $K$. Let $U=\bigcap_{i=1}^{n} U_{x_{i}}$, then $u(x) \in O$, for all $u \in U, x \in K$.

Proposition 1.2.3. A Hausdorff space $X$ is pathwise connected if and only if it is arcwise connected [8].

Proposition 1.2.4. A necessary and sufficient condition that a subset $N$ of $R^{n}$ be $n$-dimensional is that $N$ contains a non-empty subset which is open in $R^{n}$ (see [6], page 44).

Proposition 1.2.5. A subspace of a space of dimension $\leqq n$ has dimension $\leqq n$ (see [6], page 26).

Proposition 1.2.6. If $M$ is a locally compact, metric, connected space, then in order that $M$ should be locally connected, it is necessary and sufficient that for every open subset $D$ of $M$, the points of $\partial D$ that are arcwise accessible from $D$ be dense in $\partial D$ (see [7], page 106).

COROLlaRY 1.2.7. If $D$ is a domain in $R^{2}$, then the points of $\partial D$ that are arcwise accessible from $D$ are dense in $\partial D$.

PROOF. $R^{2}$ is a locally compact, connected and locally connected metric space.

Proposition 1.2.8. Every domain in $R^{2}$ is arcwise connected.

Proposition 1.2.9. If $M$ is locally compact, connected and locally connected metric space, then a necessary and sufficient condition that a point $p$ of the boundary $B$ of a domain $D$ in $M$ should be arcwise accessible from $D$ is that $p$ be accessible by closed and connected sets from $D$ (see [7], page 110).

Proposition 1.2.10. A compact connected 1-manifold is homeomorphic with a circle and a non-compact component of a 1-manifold is topologically an open interval, provided that the topology has a countable base [9].

Proposition 1.2.11. If $G$ is a connected group then $G$ is locally generated. 
Proof. We need to show that for every neighborhood $W$ of $e$ the smallest subgroup of $G$ which contains $W$ is $G$ itself.

We have the following theorem in [1] (page 37):

If $G$ is a connected group and $W$ is an open neighborhood of e, then $G=\cup_{n} W^{n}$.

If a subgroup contains $W$, it is easy to see that this subgroup contains every $W^{n}$. So, this subgroup contains $G$. That is to say that it is $G$ itself.

RemarK. A locally generated group does not need to be connected. As a matter of fact, in Enflo's paper [4], there is an example of a complete, commutative locally generated group which has more than one element and is totally disconnected.

\section{Uniform groups on connected 1-manifolds.}

\subsection{LOCALLY GENERATED, UNIFORM GROUPS WHICH ACT EFFECTIVELY AND TRAN-} SITIVELY ON THE UNIT CIRCLE.

Let $C$ denote the unit circle of the complex plane. When we study the circle $C$ we often need to talk about orientation. In this paper, we let $C$ denote the unit circle with the anticlockwise orientation.

If $f$ is a homeomorphism on $C$, then $f$ maps $C$ onto $C$ and $f$ either preserves the orientation of $C$ or reserves it. When $f$ preserves the orientation, we call $f$ an orientation preserving homeomorphism, otherwise an orientation reserving homeomorphism.

We often need to talk about an "interval" of $C$. Every two points $a, b$ of $C$ divide the circle into two intervals. In the following, we let $(a, b)$ always denote the open interval which consists of all points $z$ going from $a$ to $b$ anticlockwisely, exclusive the end points $a$ and $b$, and $[a, b]$ denote the closed interval which is the union of $(a, b)$ and the two end points $a$ and $b$.

Every homeomorphism $f$ maps an interval onto another interval. If $f$ is an orientation preserving (reserving) homeomorphism, then $f$ maps interval $(a, b)$ onto the interval $(f(a), f(b))((f(b), f(a)))$. Then we say that when $f$ is orientation preserving (reversing) on $C$, it is orientation preserving (reversing) on every interval of $C$.

We need also often to use the expression "we choose points $a$ and $b$ such that " $a<b$ "..." Then we mean that we choose a point $a$ first and then a point $b$ which comes after the point $a$ along the orientation of $C$. Of course, when " $a<b$ ", we have also " $b<a$ " in another way, but this will not make any confusion for us. If we write " $b<a$ ", then we think about point $b$ first and point $a$ comes after the point $b$ along the orientation of $C$. When we write "a $<\mathrm{c}<\mathrm{b}$ ", we mean that $c \in(a, b)$. 
Let $d(z, w)$ denote the distance between two points $z$ and $w$ along the circle. Then we have the following

Proposition 2.1.1. Assume that $G$ acts effectively as a transformation group on the unit circle $C$. Then for every $\varepsilon>0$, there is a neighborhood $U$ of e in $G$, such that for all $h \in U, z \in C$ we have $d(h(z), z)<\varepsilon$.

Proof. For every $\varepsilon>0$, we choose points $z_{i} \in C, i=1,2, \ldots, n$, such that " $z_{1}<z_{2}<\ldots<z_{n}<z_{1}$ " and $d\left(z_{i}, z_{i+1}\right)<\frac{1}{3} \varepsilon$.

Consider open intervals $\left(z_{i-1}, z_{i+2}\right), i=1,2, \ldots, n$, where we define $z_{0}=z_{n}, z_{n+1}=z_{1}, z_{n+2}=z_{2}$. Then we have $d\left(z_{i-1}, z_{i+2}\right)<\varepsilon, \forall i=1,2, \ldots, n$ and $\bigcup_{i=1}^{n}\left(z_{i-1}, z_{i+2}\right) \supset C$.

Every $\left(z_{i-1}, z_{i+2}\right)$ is open neighborhood of $\left[z_{i}, z_{i+1}\right]$. Then there exists a neighborhood $U_{i}$ of $e$ in $G$, such that $h(z) \in\left(z_{i-1}, z_{i+2}\right)$ for all $h \in U_{i}$ and for all $z \in\left[z_{i}, z_{i+1}\right]$ (see Proposition 1.2.2). Then $d(h(z), z)<\varepsilon, \forall z \in\left[z_{i}, z_{i+1}\right], h \in U_{i}$.

Let $U=\bigcap_{i=1}^{n} U_{i}$. Then $\forall h \in U$, we have $d(h(z), z)<\varepsilon, \forall z \in C$.

PROPOSITION 2.1.2. If $G$ is locally generated and acts as a transformation group on $C$, then every transformation $g \in G$ is an orientation preserving homeomorphism.

Proof. Consider a small interval $(a, b)$ of $C$. That is to say that we let $a, b$ be sufficiently close to each other. Then consider a small neighborhood $(u, v)$ of the interval $[a, b]$. Then there exists a neighborhood $U$ of $e$ in $G$ such that $U[a, b] \subset(u, v)$. If we choose $U$ sufficiently small such that $U(a)$ is sufficiently close to $a$ and $U(b)$ is sufficiently close to $b$, then every $h \in U$ maps $(a, b)$ onto $(h(a), h(b))$. This is to say that every $h \in U$ is an orientation preserving homeomorphism on $(a, b)$. So every $h \in U$ is an orientation preserving homeomorphism on C.

Since the set of all the orientation preserving homeomorphisms of $G$ constitutes a subgroup of $G$ and we assume that $G$ is locally generated, we get that every $g$ of $G$ is an orientation preserving homeomorphism.

The following proposition is inspired by Theorem 1.2.1 in Enflo [4], page 236.

Proposition 2.1.3. Let $G$ be a locally generated and uniform group, acting effectively as a transformation group on $C$ and let $M_{f}$ denote the set of fixed points for a transformation $f \in G$, where $f \neq e$. If $M_{f} \neq \emptyset$, then $\partial M_{f} \neq \emptyset$ and every point of $\partial M_{f}$ is a fixed point for all transformations of $G$.

Proof. Since $G$ acts effectively as a transformation group on $C$, we know that $M_{f} \neq C$. Then $\partial M_{f} \neq \emptyset$ when $M_{f} \neq \emptyset$. Let $x \in \partial M_{f}$. Then in every neighborhood of $x$, there is a point $x_{1}$ (which may be the same as $x$ ) such that $f\left(x_{1}\right)=x_{1}$ and $f(y) \neq y$ for all $y$ in some small interval $\left(x_{1}, x_{1}^{\prime}\right]$ or $\left[x_{1}^{\prime \prime}, x_{1}\right)$. We assume $f(y) \neq y$ for all $y$ in $\left(x_{1}, x_{1}^{\prime}\right]$ (the other case can be done analogously). 
Assume that there is a transformation $g \in G$ with $g\left(x_{1}\right) \neq x_{1}$. Then in every neighborhood of $e$ in $G$ there is an element $h$ with $h\left(x_{1}\right) \neq x_{1}$ since $G$ is locally generated.

Consider a neighborhood $\left(x_{1}^{\prime \prime}, x_{1}^{\prime}\right)$ of $x_{1}\left(x_{1}^{\prime \prime}<x_{1}<x_{1}^{\prime}\right)$, where $\left(x_{1}, x_{1}^{\prime}\right]$ satisfies the condition we mentioned above, that is to say $f(y) \neq y$ for all $y$ in $\left(x_{1}, x_{1}^{\prime}\right]$. Then there is a neighborhood $U$ of $e$ in $G$, such that $U\left(x_{1}\right) \in\left(x_{1}^{\prime \prime}, x_{1}^{\prime}\right) . G$ is uniform, so there is a neighborhood $V$ of $e$ in $G$, such that

$$
f^{n} V f^{-n}\left(x_{1}\right) \subset U\left(x_{1}\right) \subset\left(x_{1}^{\prime \prime}, x_{1}^{\prime}\right), \forall n=0,1,2, \ldots .
$$

For this $V$, there is an $h \in V$ such that $h\left(x_{1}\right) \neq x_{1}\left(h\left(x_{1}\right) \in\left(x_{1}^{\prime \prime}, x_{1}^{\prime}\right)\right)$. We assume $h\left(x_{1}\right) \in\left(x_{1}, x_{1}^{\prime}\right)$ (otherwise consider $h^{-1}$ ). T hen

$$
f^{n} h f^{-n}\left(x_{1}\right)=f^{n} h\left(x_{1}\right) \in\left(x_{1}^{\prime \prime}, x_{1}^{\prime}\right), \forall n=0,1,2, \ldots
$$

It is easy to see that $f\left(h\left(x_{1}\right)\right) \in\left(x_{1}, x_{1}^{\prime}\right)$ since $f$, as an orientation preserving homeomorphism, maps $\left[x_{1}, h\left(x_{1}\right)\right]$ onto $\left[x_{1}, f\left(h\left(x_{1}\right)\right)\right]$ and since $f$ fixes $x_{1}$ the interval $\left[x_{1}, f\left(h\left(x_{1}\right)\right)\right]$ cannot be very large when the interval $\left(x_{1}^{\prime \prime}, x_{1}^{\prime}\right)$ is sufficiently small. So $f\left(h\left(x_{1}\right)\right) \neq h\left(x_{1}\right)$.

Assume that $f\left(h\left(x_{1}\right)\right) \in\left(h\left(x_{1}\right), x_{1}^{\prime}\right)$ (otherwise consider $f^{-1}$ ).

Then $f^{n+1}\left(h\left(x_{1}\right)\right) \in\left(f^{n}\left(h\left(x_{1}\right)\right), x_{1}^{\prime}\right), \forall n=1,2, \ldots$ Let $n$ tend to infinity, then $f^{n}\left(h\left(x_{1}\right)\right)$ tends to a fixed point of $f$ in the interval $\left(x_{1}, x_{1}^{\prime}\right]$. This is a contradiction. This shows that $x_{1}$ is a fixed point for all transformations of $G$. Since $x_{1}$ can be taken arbitrarily closed to $x$, it also follows that $x$ is a fixed point for all transformations of $G$. Thus all elements of $\partial M_{f}$ are fixed points for all transformations of $G$.

COROLlaRY 2.1.4. If $G$ is locally generated, uniform and acts effectively and transitively as a transformation group on $C$. Then there is no transformation in $G$ except $e$ which has a fixed point in $C$.

Proof. If there exists an $f \in G$ except $e$, such that $f$ has fixed point on $C$, then $\partial M_{f}$ is not empty and all elements of it are fixed points for all transformations of $G$ by Proposition 2.1.3. In other words, the fixed point set of $G$ is non-empty. This is not true when $G$ acts transitively on $C$.

LeMma 2.1.5. Let $G$ be locally generated, uniform and act effectively and transitively as a transformation group on $C$.

(a) Consider any three different transformations $f, g, h \in G$. If there exists an $z \in C$ such that $f(z) \leqq g(z) \leqq h(z)$, then for any other point $z_{1} \in C$, we have $f\left(z_{1}\right) \leqq g\left(z_{1}\right) \leqq h\left(z_{1}\right)$ (Recall that $a \leqq b \leqq c$ has the precise meaning that $b \in[a, c])$.

(b) Consider any three different points $a, b, c \in C$. If $a \leqq b \leqq c$, then for every transformation $f$ of $G$, we have $f(a) \leqq f(b) \leqq f(c)$. 
Proof. (a) The interval $\left[z, z_{1}\right]$ of $C$ is homeomorphic to an interval of the real line. $f, g, h \in G$ can be seen as three increasing continuous functions on the interval since they are orientation preserving homeomorphisms on $C$. If any of the two inequalities " $f\left(z_{1}\right) \leqq g\left(z_{1}\right) \leqq h\left(z_{1}\right)$ " is not true, when " $f(z) \leqq g(z) \leqq h(z)$ " is the truth, there always exists a point $w \in C$ which is a fixed point of either $g^{-1} f \neq e$ or $h^{-1} g \neq e$. This fails by Corollary 2.1.4.

(b) By the fact that every $f$ preserves the orientation of $C$.

THEOREM 2.1.6. Let $G$ be locally generated, uniform and act effectively and transitively as a transformation group on $C$. Then $G$ is a commutative group.

Proof. To show the theorem, we need to show that for every point $z \in C$ and every two transformations $f, g \in G$, we have

$$
f g(z)=g f(z) .
$$

In order to do so, we need only to show that for all $z \in C, \varepsilon>0, f, g \in G$, we have $d(f g(z), g f(z))<\varepsilon$.

Choose an element $h \in G$ which is near $e$ but $h \neq e$ and consider the sequence $\left\{h^{i}(z), i=0,1,2, \ldots\right\}$. By Corollary 2.1.4, $h$ has no fixed point on $C$ so $h^{i}(z) \neq h^{i+1}(z)$ for all $i$.

By Proposition 2.1.1, we can choose $h$ such that $d\left[h^{i}(z), h^{i+1}(z)\right]<\frac{1}{2} \varepsilon, \forall i$.

Choose $h$ such that $z<h(z)$, that is to say, $h(z)$ comes after $z$ along the orientation of $C$ (otherwise consider $h^{-1}$ ). Then $h^{i}(z)<h^{i+1}(z), \forall i$.

Then the sequence $\left\{h^{i}(z), i=0,1,2, \ldots\right\}$ goes anticlockwisely on $C$. In other words, we have " $z<h(z)<h^{2}(z)<\ldots<h^{i}(z)<h^{i+1}(z)<\ldots$ "

If $\lim _{i \rightarrow \infty} h^{i}(z)$ exists, then $h$ has a fixed point which is a contradiction. So there is a sequence $n_{p}, p=1,2, \ldots$, such that " $h^{n_{p}}(z) \leqq z<h^{n_{p}+1}(z)$, for all $p=1,2, \ldots$ ".

Consider $f$. There is an $i \in\left\{0,1, \ldots, n_{1}\right\}$, such that $h^{i}(z) \leqq f(z) \leqq h^{i+1}(z)$. Then for any other point $z_{1} \in C$, we have $h^{i}\left(z_{1}\right) \leqq f\left(z_{1}\right) \leqq h^{i+1}\left(z_{1}\right)$ (see Lemma 2.1.5). In particular for $g(z)$ we have $h^{i}(g(z)) \leqq f(g(z)) \leqq h^{i+1}(g(z))$.

Similarly, for $g$, there is $j \in\left\{1,2, \ldots, n_{1}\right\}$, such that $h^{j}(z) \leqq g(z) \leqq h^{j+1}(z)$, and for $f(z)$ we have $h^{j}(f(z)) \leqq g(f(z)) \leqq h^{j+1}(f(z))$.

Since $g(z) \in\left[h^{j}(z), h^{j+1}(z)\right]$, we have $h^{i}(g(z)) \in\left[h^{i+j}(z), h^{i+j+1}(z)\right]$ and $h^{i+1}(g(z)) \in$ $\left[h^{i+j+1}(z), h^{i+j+2}(z)\right]$ (see Lemma 2.1.5).

Then $f(g(z)) \in\left[h^{i}(g(z)), h^{i+1}(g(z))\right] \subset\left[h^{i+j}(z), h^{i+j+2}(z)\right]$.

Similarly, we can prove that $g(f(z)) \in\left[h^{i+j}(z), h^{i+j+2}(z)\right]$.

These results give that $d(f g(z), g f(z))<\varepsilon$.

\subsection{CONNECTED, UNIFORM GROUPS ON CONNECTED 1-MANIFOLDS}

Proposition 1.2.10 tells us that a compact connected 1-manifold is homeomorphic with the unit circle and a non-compact component is topologically an open interval, provided it is countable (i.e., there is a countable base for the topology), 
so a connected and countable 1-manifold is topologically either a circle or the real line $R$.

In Enflo [4], there is the following

THEOREM 2.2.1. If a locally generated, uniform group $G$ acts effectively as a transformation group on the real line $R$, then it is commutative.

We have shown in Proposition 1.2.11 that a connected group is locally generated, so we have the following

COROLLARY 2.2.2. If a connected, uniform group $G$ acts effectively as a transformation group on the real line $R$, then it is commutative.

THEOREM 2.2.3. If a connected and uniform group $G$ acts effectively as a transformation group on the unit circle $C$, then it is commutative.

Proof. If there exists a point $c$ of $C$ such that $c$ is a fixe point of all transformations of $G$, then $G$ is a connected and uniform group, acts effectively as a transformation group on $C-\{c\}$ which is homeomorphic with the real line $R$. Then by Corollary 2.2.2, $G$ is commutative on $C-\{c\}$. Then it is easy to see that $G$ is commutative on $C$. In other words, the theorem is proved in case the set of fixed points for all transformations of $G$ is not the empty set.

We study the orbits $G(z)$ of $G$, where $z \in C$. We know that $G$ acts transitively as a transformation group on every orbit $G(z)$.

By Proposition 1.2.1, an orbit $G(z)=\{g(z), g \in G\}$ is a connected subset of $C$ when $G$ is connected. We have one of the following two cases: (1) $G(z)=C,(2)$ $G(z) \neq C$.

In case (1) when $G(z)=C, G$ is a locally generated (since it is connected) and uniform group, acts effectively and transitively as a transformation group on $C$. $G$ is commutative by Theorem 2.1.6.

In case (2) when $G(z) \neq C$, it is easy to see that $G(z)$, since it is connected, is in one of the following three cases: (a) $G(z)$ is a single point, (b) $G(z)$ is a proper interval of $C$, open, closed or half closed, (c) $G(z)$ is $C$ except one point. In case (b) we actually also know that $G(z)$ is an open interval since for every two points of an orbit, there is always a homeomorphism $g$ which maps one point to the other. So if there is one point which is an inner point, every other point is an inner point.

In case (a), it is easy to see that the single point of the orbit is a fixed point for all $g \in G$. In case (c), the single point remained by the orbit is a fixed point for all of $g \in G$. In case (b), it is easy to show that the end points of the interval are fixed points for all $g \in G$.

In all the three cases, the set of fixed points for all transformations of $G$ is not empty. The theorem is proved. 
COROLlaRY 2.2.4. If a connected and uniform group $G$ acts effectively as a transformation group on a connected and countable 1-manifold, then it is commutative.

\section{Uniform transformation groups on $R^{2}$.}

In this chapter we study uniform transformation groups on $R^{2}$. The main theorem is the following:

Assume $G$ be a uniform group which acts openly as a transformation group on $R^{2}$. Let $x_{0} \in R^{2}$ and $G_{0}$ be the subgroup of $G$, which consists of all the transformations that have $x_{0}$ as a fixed point. Assume that $G_{0}$ is arcwise connected. Then if $G_{0}$ acts effectively on $R^{2}, G_{0}$ is a commutative subgroup of $G$.

In order to prove this main theorem, we prove, under the same assumptions on $G$ and $G_{0}$ but without that $G_{0}$ acts effectively on $R^{2}$, the following results about the orbits of points under $G_{0}$ and the set of fixed points of $G_{0}$ :

(a) If $G_{0}$ does not leave every point of $R^{2}$ fixed, then there is an open, connected and dense subset $O$ of $R^{2}$, such that, for every point $x$ of $O$, the orbit $G_{0} x$ is either a Jordan curve or homeomorphic with the real line R. Each $G_{0} x$ is closed so if it is homeomorphic with the real line, it is unbounded. (b) If $G_{0}$ does not leave every point of $R^{2}$ fixed, then it fixes at most two points of $R^{2}$. (c) In case there are exactly two fixed points of $G_{0}$, for every point $x$ of $R^{2}$ other than the two fixed points, the orbit $G_{0} x$ is either a Jordan curve or homeomorphic with the real line.

\subsection{THE SETS $M_{\delta}$ AND THEIR BOUNDARIES $\partial M_{\delta}$}

The following assumptions on $G$ and $G_{0}$ will be in force throughout Chapter 3 . Assume that $G$ is a uniform group that acts openly as a transformation group on $R^{2}$. Let $x_{0} \in R^{2}$ and $G_{0}$ be the subgroup of $G$, which consists of all the transformations that have $x_{0}$ as fixed point. Suppose that $G_{0}$ arcwise connected.

For $\delta>0$, let $B_{\delta, x_{0}}$ denote the open ball which has $x_{0}$ as its center and $\delta$ as its radius. Consider the subsets of $R^{2}$, which are in the form:

$$
M_{\delta}=\bigcup_{g \in G_{0}} g\left(B_{\delta, x_{0}}\right) \text {. }
$$

Then every $M_{\delta}$ is an open neighborhood of $x_{0}$. The sets $M_{\delta}$ and their boundaries $\partial M_{\delta}$, for all $\delta>0$, play an important role in this chapter. We have the following propositions:

Proposition 3.1.1. $\left\{M_{\delta}\right\}_{\delta>0}$ is a base for the neighborhood system of $x_{0}$ in $R^{2}$.

Proof. Given $\varepsilon>0$, there is a neighborhood $U$ of $e$ in $G$, such that,

$$
g U g^{-1}\left(x_{0}\right) \subseteq B_{\varepsilon, x_{0}},
$$


for all $g \in G$, since $G$ is uniform. Especially for all $g \in G_{0}$, (1) holds and has form

$$
g U x_{0} \subseteq B_{\varepsilon, x_{0}} .
$$

For this $U$, since $G$ acts openly on $R^{2}, U x_{0}=\left\{h\left(x_{0}\right), h \in U\right\}$ is a neighborhood of $x_{0}$. Then there is an $\delta>0$, such that

$$
B_{\delta, x_{0}} \subseteq U x_{0}
$$

and therefore

$$
g\left(B_{\delta, x_{0}}\right) \subseteq g U x_{0} \subseteq B_{\varepsilon, x_{0}}
$$

for all $g \in G_{0}$. Then

$$
\bigcup_{g \in G_{0}} g\left(B_{\delta, x_{0}}\right) \subseteq B_{\varepsilon, x_{0}} .
$$

This is to say

$$
M_{\delta} \subseteq B_{\varepsilon, x_{0}} .
$$

In other words, we have shown that given $\varepsilon>0$, there is $\delta>0$, such that $M_{\delta} \subseteq B_{\varepsilon, x_{0}}$. This gives that $\left\{M_{\delta}\right\}_{\delta>0}$ is a base for the neighborhood system of $x_{0}$.

PROPOSITION 3.1.2. (a) Every $M_{\delta}$ is invariant under the transformation subgroup $G_{0}$, that is to say: $G_{0} M_{\delta}=M_{\delta}$.

(b) Also the closure $\bar{M}_{\delta}$ is invariant under the transformation subgroup $G_{0}: G_{0} \bar{M}_{\delta}=\bar{M}_{\delta}$.

(c) Also the boundary of $M_{\delta}: \partial M_{\delta}=\bar{M}_{\delta} \backslash M_{\delta}$, is invariant under the transformation subgroup $G_{0}: G_{0}\left(\partial M_{\delta}\right)=\partial M_{\delta}$.

PROOF. The proofs are trivial.

Proposition 3.1.3. For every two $\delta$ and $\delta^{\prime}$ with $0<\delta^{\prime}<\delta$, we have $\bar{M}_{\delta^{\prime}} \subset M_{\delta}$.

Proof. It is trivial to show that if $0<\delta^{\prime}<\delta$, then $M_{\delta^{\prime}} \subset M_{\delta}$. Let $x \in \partial M_{\delta^{\prime}}$. We show that $x \in M_{\delta}$.

Since $x \in \partial M_{\delta^{\prime}}$, there is a sequence $x_{n} \in M_{\delta^{\prime}}$ such that $x_{n} \rightarrow x$. The sequence $x_{n}$ can be written as $h_{n}\left(z_{n}\right)$ with $z_{n} \in B_{\delta^{\prime}, x_{0}}$ and $h_{n} \in G_{0}$. Then there is a subsequence of $z_{n}$ which converges to a point $z \in \bar{B}_{\delta^{\prime}, x_{0}}$. Without loosing the generality, assume that $z_{n} \rightarrow z$. This gives that $h_{n}^{-1}\left(x_{n}\right) \rightarrow z \in \bar{B}_{\delta^{\prime}, x_{0}}$.

Consider a small open neighborhood $U$ of $e$ in $G$. Then $U z$ and $U x$ are small open neighborhoods of $z$ and $x$ respectively in $R^{2}$. For this $U$, there is an open neighborhood $V$ of $e$ in $G$ such that $f V f^{-1} \subset U$ for all $f \in G$. Then $V z$ and $V x$ are also small open neighborhoods of $z$ and $x$ respectively in $R^{2}$. That $x_{n} \rightarrow x$ gives that $x_{n} \in V x$ and that $h_{n}^{-1}\left(x_{n}\right) \rightarrow z$ gives that $h_{n}^{-1}\left(x_{n}\right) \in U z$ when $n$ is sufficiently large. Then we see that $h_{n}^{-1}\left(x_{n}\right) \in h_{n}^{-1} V x \subset U h_{n}^{-1}(x)$, which gives that $h_{n}^{-1}(x) \in U^{-1} h_{n}^{-1}\left(x_{n}\right) \subset U^{-1} U(z)$ when $n$ is sufficiently large. 
We see that $U^{-1} U(z)$ is a small open neighborhood of $z$ in $R^{2}$ when $U$ is a small open neighborhood of $e$ in $G$. So when $U$ is sufficiently small, we have that $h_{n}^{-1}(x) \in U^{-1} U(z) \subset B_{\delta, x_{0}}$, which gives that $x \in M_{\delta}$. The proposition is proved.

Proposition 3.1.4. For every $\delta>0$, we have (a) $\operatorname{dim} M_{\delta}=2$; (b) if $\partial M_{\delta}$ is not empty, then either $\operatorname{dim} \partial M_{\delta}=0$ or $\operatorname{dim} \partial M_{\delta}=1$.

Proof. (a) $\operatorname{dim} M_{\delta}=2$, since $M_{\delta}$ is an open subset of $R^{2}$ (see Proposition 1.2.4).

(b) $\partial M_{\delta}$ is a subset of $R^{2}$ so $\operatorname{dim} \partial M_{\delta} \leqq 2$ (see Proposition 1.2.5). But $\operatorname{dim} \partial M_{\delta} \neq 2$ since $\partial M_{\delta}$ does not contain a non-empty subset which is open in $R^{2}$. Thus $\operatorname{dim} \partial M_{\delta} \leqq 1$ from which (b) follows directly.

So the boundary $\partial M_{\delta}$ is either 0-dimensional or 1-dimensional. $G_{0}$ is a uniform transformation group on $\partial M_{\delta}$. In chapter 2 , we have studied uniform transformation groups on connected and countable 1-manifold. It is natural to hope that every $\partial M_{\delta}$ consists of either connected 0 -manifolds or connected 1-manifolds. If so, $G_{0}$ will be commutative on every $\partial M_{\delta}$ by using the results of Chapter 2 . After this, if the points of the sets $\partial M_{\delta}$ for $\delta>0$ fill up all of $R^{2}$, then $G_{0}$ will be commutative on $R^{2}$.

In this section, we give first a proof that the sets $\partial M_{\delta}$ for $\delta>0$ fill up all of $R^{2}$ indeed in the following

Proposition 3.1.5. For every point $x$ of $R^{2}$ other than $x_{0}$, there is a unique $\delta>0$ such that $x \in \partial M_{\delta}$. Moreover, for this $\delta$, there exists a sequence $g_{n}(x) \in G_{0} x$ which converges to a point $y \in \partial B_{\delta, x_{0}}$. In particular, if $x$ is a fixed point of $G_{0}$ then $x \in \partial B_{\delta, x_{0}}$.

In the proof of Proposition 3.1.5, we use the following fact which we present as a lemma.

LEMMA 3.1.6. Suppose that $x$ and $y$ are points of $R^{2}$ and $g_{n}$ is a sequence of $G_{0}$. Then $g_{n}(x) \rightarrow y$ if and only if $g_{n}^{-1}(y) \rightarrow x$.

Proof. That $G$ is a uniform group gives that for every open neighborhood $U$ of $e$ in $G$, there exists an open neighborhood $V$ of $e$ in $G$ such that $g^{-1} V g \subset U$, $\forall g \in G$. We may choose $V$ such that $V=V^{-1}$. Assume that $g_{n}(x) \rightarrow y$. Since $G$ acts openly as a transformation group on $R^{2}$, we have that $g_{n}(x) \in V y$ when $n \rightarrow \infty$. This gives that $y \in V^{-1} g_{n}(x)=V g_{n}(x)$. Then we have $g_{n}^{-1}(y) \in$ $g_{n}^{-1} V g_{n}(x) \subset U x$. This is to say that $g_{n}^{-1}(y) \rightarrow x$. In the same way, we can show that $g_{n}(x) \rightarrow y$ if $g_{n}^{-1}(y) \rightarrow x$.

Proof of Proposition 3.1.5. Since $x \neq x_{0}$, we see that $x \notin M_{\gamma}$ when $\gamma$ is sufficiently small by Proposition 3.1.1. Let $\delta=\sup \left\{\gamma, \mathrm{x} \notin M_{\gamma}\right\}$. Since when $\gamma$ is sufficiently large we have $x \in B_{\gamma, x_{0}}=e\left(B_{\gamma, x_{0}}\right) \subset \bigcup_{g \in G_{0}} g\left(B_{\gamma, x_{0}}\right)=M_{\gamma}$, we see that 
$0<\delta<\infty$. Besides, since $M_{\gamma_{1}} \subset M_{\gamma_{2}}$ when $0<\gamma_{1}<\gamma_{2}$, we see that for every $\gamma$ with $0<\gamma<\delta$, we have $x \notin M_{\gamma}$. Let us show that $x \in \partial M_{\delta}$.

If $x \in M_{\delta}$, then there is a $g \in G_{0}$, such that $x \in g\left(B_{\delta, x_{0}}\right)$. Then there is a $\gamma$ with $0<\gamma<\delta$ such that $x \in g\left(B_{\gamma, x_{0}}\right) \subseteq M_{\gamma}$. This is a contradiction. So $x \notin M_{\delta}$. We need only to show that $x \in \bar{M}_{\delta}$.

For every $\gamma>\delta$, we have $x \in M_{\gamma}$. Then there is $g^{-1} \in G_{0}$, such that $x \in g^{-1}\left(B_{\gamma, x_{0}}\right)$. Then $g(x) \in B_{\gamma, x_{0}}$.

This gives that there is a sequence $g_{n}$ of $G_{0}$, such that $g_{n}(x)$ converges to a point $y \in \partial B_{\delta, x_{0}}$. This is equivalent to $g_{n}^{-1}(y) \rightarrow x$ by the Lemma 3.1.6. But $g_{n}^{-1}(y) \in G_{0} y \subset G_{0}\left(\partial B_{\delta, x_{0}}\right) \subset G_{0}\left(\bar{M}_{\delta}\right)=\bar{M}_{\delta}$, we have $x \in \bar{M}_{\delta}$.

We have proved $x \in \partial M_{\delta}$, and also the existence of the sequence $g_{n}(x) \in G_{0} x$ such that $g_{n}(x) \rightarrow y$, with $y \in \partial B_{\delta, x_{0}}$. When $x$ is a fixed point of $G_{0}$, it is easy to see that $y=x \in \partial B_{\delta, x_{0}}$.

This $\delta$ is unique for $x$ with respect to the property that $x \in \partial M_{\delta}$. In fact, if we consider another value $\gamma$ with $\gamma>\delta$ then $x \in M_{\gamma}$ so $x \notin \partial M_{\gamma}$. If $0<\gamma<\delta$ we have also $x \notin \partial M_{\gamma}$ by Proposition 3.1.3, since $x \in \partial M_{\delta}$. Proposition 3.1 .5 is proved.

It is desirable that every $\partial M_{\delta}$ only consists of either connected 0 -manifolds or connected 1-manifolds. To prove this, we need to study the sets $\partial M_{\delta}$ in detail. One of the key tools turns out to be the following proposition which is crucial for our proofs:

Proposition 3.1.7. For every $\delta>0, \partial M_{\delta}$ is arcwise accessible from $M_{\delta}$.

To prove Proposition 3.1.7, we need the following

LEMMA 3.1.8. If $x \in \partial M_{\delta}$ is arcwise accessible from $M_{\delta}$, then $\overline{G_{0} x}$, as a subset of $\partial M_{\delta}$, is arcwise accessible from $M_{\delta}$.

Proof. It is easy to show that if $x \in \partial M_{\delta}$ and $x$ is arcwise accessible from $M_{\delta}$, then the orbit $G_{0} x$, as a subset of $\partial M_{\delta}$, is arcwise accessible from $M_{\delta}$. In fact, when $x \in \partial M_{\delta}$ and $x$ is arcwise accessible from $M_{\delta}$, there is an arc $T$ from a point $a \in M_{\delta}$ to $x$ such that $T-x \in M_{\delta}$. Every element $g$ of $G_{0}$ is a homeomorphism of $R^{2}$ to $R^{2}$, so it maps arc $T$ to an $\operatorname{arc} g(T)$. Since both $M_{\delta}$ and $\partial M_{\delta}$ are invariant under $G_{0}$ we see that $g(T-x) \in M_{\delta}$ and $g(x) \in \partial M_{\delta}$. This gives that $g(x)$ is arcwise accessible from $M_{\delta}$.

To show that even the closure $\overline{G_{0} x}$ of the orbit $G_{0} x$, which is also a subset of $\partial M_{\delta}$, is arcwise accessible from $M_{\delta}$, we need to show that if $g_{n}(x) \rightarrow z$, where $g_{n} \in G_{0}$, then $z$ is arcwise accessible from $M_{\delta}$. By Proposition 1.2.9, it is enough to show that $z$ is accessible by a closed and connected set $K$ from $M_{\delta}$.

Since $x \in \partial M_{\delta}$ is arcwise accessible from $M_{\delta}$, there is an $\operatorname{arc} T$ from a point of $M_{\delta}$ to $x$ such that $T-x \subset M_{\delta}$. Suppose $g_{n}(x) \rightarrow z$. Then for every $t \in T, g_{n}(t)$ is 
a sequence of $R^{2}$. We say that if we choose $T$ sufficiently closed to $x$, then $\left\{g_{n}(T), \forall n\right\}$ is bounded.

In fact, since $G$ is uniform, for every open neighborhood $U$ of $e$ in $G$, there is an open neighborhood $V$ of $e$ in $G$ such that $g_{n} V g_{n}^{-1} \subset U$ for all $n$. This gives $V \subset g_{n}^{-1} U g_{n}$ for all $n$. Then $T \subset V x \subset g_{n}^{-1} \cup g_{n}(x)$ for all $n$ if $T$ is sufficiently close to $x$. This gives $g_{n}(T) \subset U g_{n}(x)$. But $g_{n}(x) \rightarrow z$, so $g_{n}(x) \in U z$ when $n \rightarrow \infty$. Then we get $g_{n}(T) \subset U U z$. $U U z$ is a small open neighborhood of $z$ when $U$ is a small open neighborhood of $e$.

Then every sequence $g_{n}(t)$ has at least one limit point in $R^{2}$ for $t \in T$.

Let $K$ be the set which consists of all limit points of $g_{n}(t)$ with $t \in T$. Then $z \in K$. We show that $K$ is closed, connected and $K-z \subset M_{\delta}$.

Let us first show that $K-\mathrm{z} \subset M_{\delta}$. For this, it is enough to show that if $t \in T-x \subset M_{\delta}$, then any limit point of $g_{n}(t)$ is in $M_{\delta}$. If it is not the truth, there is a sequence $g_{n_{k}}(t) \rightarrow w \in \partial M_{\delta}$. This gives $g_{n_{k}}^{-1}(w) \rightarrow t$. But $g_{n_{k}}^{-1}(w) \in \partial M_{\delta}$ since $\partial M_{\delta}$ is invariant under $G_{0}$, so $t \in \partial M_{\delta}$. This is a contradiction.

Next, we show that $K$ is closed.

Let $a$ be a limit point of a sequence of points of $K$. That is to say, let $a_{m} \rightarrow a$, where every $a_{m}$ is a limit point of $g_{n}\left(t_{m}\right)$, with $t_{m} \in T$. Then it is easy to see that there is a sequence $g_{n_{m}}\left(t_{m}\right)$ converging to $a$ when $m \rightarrow \infty$ (then also $n_{m} \rightarrow \infty$ ). $t_{m}$ has a convergent subsequence. Without loosing the generality, let $t_{m} \rightarrow t \in T$. Study the sequence $g_{n_{m}}(t)$. This sequence has a limit point $a^{\prime}$. Without loosing the generality, suppose $g_{n_{m}}(t) \rightarrow a^{\prime}$. Then $a^{\prime} \in K$.

Let $U$ and $V$ be two arbitrary open neighborhoods of $e$ in $G$ such that $V V \subset U$. Then $V a^{\prime}$ is an open neighborhood of $a^{\prime}$ in $R^{2}$. Then $g_{n_{m}}(t) \in V a^{\prime}$ when $m$ is large. On the other hand, since $t_{m} \rightarrow t$ and $G$ is a uniform group, we have $t_{m} \in g_{n_{m}}^{-1} V g_{n_{m}}(t)$ when $m$ is large. This gives $g_{n_{m}}\left(t_{m}\right) \in V g_{n_{m}}(t) \subset V V a^{\prime} \subset U a^{\prime}$. That is to say $g_{n_{m}}\left(t_{m}\right) \rightarrow a^{\prime}$. But $g_{n_{m}}\left(t_{m}\right) \rightarrow a$, so $a=a^{\prime}$. This gives $a \in K$. We have proved that $K$ is closed.

Finally, we show that $K$ is connected.

Let $K=K_{1} \cup K_{2}, K_{1}, K_{2}$ are nonempty and $K_{1} \cap K_{2}=\emptyset$. We show that one of $\bar{K}_{1} \cap K_{2}$ and $K_{1} \cap \bar{K}_{2}$ be nonempty. Without loosing the generality, we suppose $z \in K_{2}$.

Let $T_{1}$ be the subset of $T$ consisting of such $t$ so that some limit point of $g_{n}(t)$ is in $K_{1}, T_{2}$ be the subset of $T$ consisting of such $t$ so that none of the limit points of $g_{n}(t)$ be in $K_{1}$. Then $T_{1} \cup T_{2}=T$ with $T_{1} \cap T_{2}=\emptyset$. Besides, it is easy to see that none of $T_{1}$ or $T_{2}$ be empty $\left(z \in T_{2}\right)$.

For every $t \in T_{2}$, a subsequence of $g_{n}(t)$ can only converge to a point of $K_{2}$.

Since none of $T_{1}$ or $T_{2}$ be empty and $T$ is connected, we know that one of $\bar{T}_{1} \cap T_{2}$ and $T_{1} \cap \bar{T}_{2}$ is nonempty.

When $\bar{T}_{1} \cap T_{2}$ is nonempty, let $t$ be a common point of them. Then there is a sequence $t_{m} \rightarrow t$ with $t_{m} \in T_{1}$. Then for every $m$ there exists a subsequence of 
$g_{n}\left(t_{m}\right)$ converging to $b_{m} \in K_{1}, b_{m}$ has a limit point $b$. Without loosing the generality we suppose $b_{m} \rightarrow b$. Then $b \in \bar{K}_{1}$. On the other hand, as we have shown before, there exists a sequence $g_{n_{m}}\left(t_{m}\right)$ converging to $b$. For this sequence $g_{n_{m}}$ we know that even $g_{n_{m}}(t)$ converges to $b$. This is to say that $b$ is a limit point of sequence $g_{n}(t)$. But $t \in T_{2}$, so $b \in K_{2}$. This shows that $\bar{K}_{1} \cap K_{2} \neq \emptyset$.

When $T_{1} \cap \bar{T}_{2}$ is nonempty, let $t$ be a common point of them. Since $t \in T_{1}$, there exists a subsequence $g_{n_{k}}(t) \rightarrow b \in K_{1}$. On the other hand, there is a sequence $t_{m} \rightarrow t, t_{m} \in T_{2}$. For every $m$, consider sequence $g_{n_{k}}\left(t_{m}\right)$. Then there is a subsequence of $g_{n_{k}}\left(t_{m}\right)$ which converges to some $b_{m} \in K_{2} . b_{m}$ has a limit point $b^{\prime}$. Without loosing the generality, suppose $b_{m} \rightarrow b^{\prime}$. Then $b^{\prime} \in \bar{K}_{2}$. It is easy to see that there is a sequence $g_{n_{k_{m}}}\left(t_{m}\right)$ converging to $b^{\prime}$ and then even the sequence $g_{n_{k_{m}}}(t)$ converges to $b^{\prime}$, when $m \rightarrow \infty$. Then we see that $b=b^{\prime}$. This shows that $K_{1} \cap \bar{K}_{2} \neq \emptyset$. We have proved that $K$ is connected.

Therefore, we have proved that $\overline{G_{0} x}$ is arcwise accessible from $M_{\delta}$.

Proof of Proposition 3.1.7. If $x \in \partial M_{\delta}$ we have proved in Proposition 3.1.5 that there exists a sequence $g_{n}(x) \in G_{0} x$ which converges to a point $y \in \partial B_{\delta, x_{0}}$. Then $y \in \overline{G_{0} x}$. This gives also $x \in \overline{G_{0} y}$ by Lemma 3.1.6.

It is easy to see that $y$ is arcwise accessible from $M_{\delta}$ since $y$ is arcwise accessible from $B_{\delta, x_{0}}$. So $x$ is also arcwise accessible from $M_{\delta}$ by Lemma 3.1.8.

For the further investigations of the sets $\partial M_{\delta}$, we need to study the complement of the sets $\bar{M}_{\delta}$ in $R^{2}$. We do this in the next section of this chapter.

\subsection{THE SETS $A_{\delta}$ AND THEIR BOUNDARIES $\partial A_{\delta}$.}

For every $\delta>0$, the set $M_{\delta}$ is open and connected. The closure $\bar{M}_{\delta}$ is closed and connected. The complement of $\bar{M}_{\delta}$ in $R^{2}$, if it is not the empty set, is a disjoint union of its components. Let $A_{\delta}$ denote an arbitrary component of $\bar{M}_{\delta}^{c}$ in $R^{2}$. Then $A_{\delta}$ is open and connected. We study the set $A_{\delta}$ and its boundary $\partial A_{\delta}$.

Proposition 3.2.1. $\partial A_{\delta}$ is a subset of $\partial M_{\delta}$.

Proposition 3.2.2. (a) $A_{\delta}$ is invariant under $G_{0}$; (b) $\bar{A}_{\delta}$ is invariant under $G_{0}$; (c) $\partial A_{\delta}$ is invariant under $G_{0}$.

Proof. (a) Consider a point $x \in A_{\delta}$. If there is an $g \in G_{0}$, such that $g(x) \notin A_{\delta}$, then $g(x)$ is in another component $A_{\delta}^{\prime}$ of $\bar{M}_{\delta}^{c}$ since $\bar{M}_{\delta}$ is invariant under $G_{0}$. Then there is a path $g_{t}(x), t \in[0,1], g_{t} \in G_{0}$, which goes from $x$ to $g(x)$ since $G_{0}$ is arcwise connected. This path must intersect the boundary of $A_{\delta}$. Then there is an $t$ such that $g_{t}(x) \in \partial A_{\delta} \subset \partial M_{\delta}$. This gives that $x \in \bar{M}_{\delta}$. This is a contraction. (a) is proved. The proofs of $(b)$ and $(c)$ are trivial.

Proposition 3.2.3. If $x \in \partial A_{\delta}$ and $x$ is arcwise accessible from $A_{\delta}$ then the orbit $G_{0} x$, as a subset of $\partial A_{\delta}$, is arcwise accessible from $A_{\delta}$. 
Proof. The proof is similar the first part of the proof of Lemma 3.1.8.

We consider orbits $G_{0} x$ for $x \in \partial A_{\delta}$. Then every $\operatorname{dim} G_{0} x \leqq 1$, since $G_{0} x$ is a subset of $\partial M_{\delta}$. Thus, either $\operatorname{dim} G_{0} x=0$ or $\operatorname{dim} G_{0} x=1$.

Proposition 3.2.4. For every $x \in \partial A_{\delta}$, if $\operatorname{dim} G_{0} x=1$ and $x$ is arcwise accessible from $A_{\delta}$, then $G_{0} x$, provided with the relative topology inherited from $R^{2}$, is a 1-manifold.

Proof. Since $G_{0}$ is arcwise connected, for every two points $p$ and $q$ of $G_{0} x$ (that the orbit is 1-dimensional gives that at least two points of the orbit exist) there is an path $g_{t}(p), t \in[0,1]$ of $G_{0} x$ which goes from $p$ to $q$ with $g_{0}(p)=p, g_{1}(p)=q$. This implies that there exists an arc $\overline{p q}$ of $G_{0} x$ which goes from $p$ to $q$. We consider an interior point $d$ of the arc $\overline{p q}$. Since $G_{0} x=G_{0} d$, without loosing the generality, we choose $x$ as an interior point of the arc $\overline{p q}$. Without loosing the generality, we also choose $p$ and $q$ such that the arc $\overline{p q}$ is close to $x$.

Since $x$ is arcwise accessible from $A_{\delta}$, both $p$ and $q$ are arcwise accessible from $A_{\delta}$. Then there are two arcs $T_{1}$, resp. $T_{2}$ from some common point of $A_{\delta}$ to $p$ resp. $q$ such that $T_{1}-p, T_{2}-q \subset A_{\delta}$. We may choose $T_{1}$ and $T_{2}$ such that $T_{1} \cup \overline{p q} \cup T_{2}$ constitute a Jordan curve in $R^{2}$. We call this Jordan curve $C_{1}$.

By the Jordan curve theorem, $C_{1}$ separates $R^{2}$ into two connected and open sets $\mathscr{D}_{1}$ and $\mathscr{D}_{2}$, one bounded and the other unbounded, and $C_{1}$ is the boundary of each.

We claim that $M_{\delta}$ can only intersect one of $\mathscr{D}_{1}$ and $\mathscr{D}_{2}$. In fact, if this is not true, there are two points of $M_{\delta}$, one in $\mathscr{D}_{1}$ and the other in $\mathscr{D}_{2} . M_{\delta}$ is a domain of $R^{2}$ so there is an arc of $M_{\delta}$ from the one point to the other (see Proposition 1.2.8). This arc must intersect the Jordan curve $C_{1}$. But $C_{1}$ consists only of points of $A_{\delta}$ and points of $\partial A_{\delta}$ which is a part of $\partial M_{\delta}$. An arc of $M_{\delta}$ cannot have any common point with $C_{1}$. This contradiction shows that $M_{\delta}$ intersect only one of $\mathscr{D}_{1}$ and $\mathscr{D}_{2}$.

Let $\mathscr{D}_{1}$ be the one which does not intersect $M_{\delta}$. Then there is no point of $\partial M_{\delta}$ in $\mathscr{D}_{1}$. In fact, if there is a point of $\partial M_{\delta}$ in $\mathscr{D}_{1}$, then $\mathscr{D}_{1}$ is an open neighborhood of the point. Then there is a point of $M_{\delta}$ in $\mathscr{D}_{1}$. This is a contradiction. This gives that there is no point of $G_{0} x$ in $\mathscr{D}_{1}$.

But $\mathscr{D}_{1}$ contains points of $A_{\delta}$ since part of the boundary of $\mathscr{D}_{1}$ is in $A_{\delta}$ and $A_{\delta}$ is open. Let us show that $\mathscr{D}_{1}$ contains only points of $A_{\delta}$. If this is not true, there is point of $\mathscr{D}_{1}$, which is not a point of $A_{\delta}$. $\mathscr{D}_{1}$ is a domain of $R^{2}$. If we choose, in $\mathscr{D}_{1}$, a point of $A_{\delta}$ and a point which is not in $A_{\delta}$, there is an arc of $\mathscr{D}_{1}$ joining the two points. This arc must intersect the boundary of $A_{\delta}$. Then we have found a point of $\partial M_{\delta}$ in $\mathscr{D}_{1}$. This is a contradiction.

On the other hand, the point $x$ is arcwise accessible from $M_{\delta}$ by Proposition 3.1.7 since $x$ is a point of $\partial M_{\delta}$. Then both $p$ and $q$ are arcwise accessible from $M_{\delta}$. 
So there are two $\operatorname{arcs} L_{1}$, resp. $L_{2}$ from some point of $M_{\delta}$ to $p$ resp. $q$, such that $L_{1}-p, L_{2}-q \subset M_{\delta}$. We can choose $L_{1}$ and $L_{2}$ such that $L_{1} \cup \overline{p q} \cup L_{2}$ constitute a Jordan curve in $R^{2}$. We call this Jordan curve $C_{2}$.

By Jordan curve theorem, $C_{2}$ also separates $R^{2}$ into two connected and open sets $\mathscr{B}_{1}$ and $\mathscr{B}_{2}$, one bounded and the other unbounded, and $C_{2}$ is the boundary of each.

We claim that $A_{\delta}$ can only intersect one of $\mathscr{B}_{1}$ and $\mathscr{B}_{2}$. If there are two points of $A_{\delta}$, one is in $\mathscr{B}_{1}$ and the other is in $\mathscr{B}_{2}$, then there is an arc of $A_{\delta}$ joining the two points since $A_{\delta}$ is a domain of $R^{2}$. This arc must intersect the Jordan curve $C_{2}$. But $C_{2}$ consists only of points of $M_{\delta}$ and points of $\partial A_{\delta}$ which is a part of $\partial M_{\delta}$. An arc of $A_{\delta}$ has no common point with $C_{2}$.

Let $\mathscr{B}_{1}$ be the one which does not intersect $A_{\delta}$. Then no point of $\partial A_{\delta}$ is in $\mathscr{B}_{1}$. In fact, if there is a point of $\partial A_{\delta}$ in $\mathscr{B}_{1}$, then $\mathscr{B}_{1}$ is an open neighborhood of the point. Then there is a point of $A_{\delta}$ in $\mathscr{B}_{1}$. This is a contradiction. This gives that no point of $G_{0} x$ is in $\mathscr{B}_{1}$.

It is easy to see that we have $\mathscr{D}_{1} \subset \mathscr{B}_{2}$ and $\mathscr{B}_{1} \subset \mathscr{D}_{2}$.

We have supposed that $x$ is an interior point of the arc $\overline{p q}$. Then it is easy to see that $\mathscr{D}_{1} \cup \overline{p q} \cup \mathscr{B}_{1}$ is an open neighborhood of $x$ in $R^{2}$ in which there are no more points of $G_{0} x$ other than the $\operatorname{arc} \overline{p q}$. This proves that the orbit $G_{0} x$, at the point $x$, provided with the relative topology inherited from $R^{2}$, is locally an arc.

For any two points of the orbit $G_{0} x$, there is a homeomorphism $g \in G_{0}$ which send the one point to the other. If the orbit $G_{0} x$ is locally an arc at one point, provided with the relative topology inherited from $R^{2}$, then it is locally an arc at every point of it, provided with the relative topology inherited from $R^{2}$. This gives that $G_{0} x$ is an 1-manifold, provided with the relative topology inherited from $R^{2}$.

CoROllary 3.2.5. For every $x \in \partial A_{\delta}$, if $\operatorname{dim} G_{0} x=1$ and $x$ is arcwise accessible from $A_{\delta}$, then there is a neighborhood of $x$ in which there are no more points of $\partial A_{\delta}$ other than an arc of $G_{0} x$. In particular, the orbit $G_{0} x$ is open in $\partial A_{\delta}$.

Proof. See the proof of Proposition 3.2.4.

Corollary 3.2.6. For every $x \in \partial A_{\delta}$, if $\operatorname{dim} G_{0} x=1$ and $x$ is arcwise accessible from $A_{\delta}$, then $G_{0} x$ is closed.

Proof. To show that $G_{0} x$ is closed, we need only to show that $\partial G_{0} x \subset G_{0} x$. If this is not true, there is a sequence $g_{n}(x) \rightarrow y \in \partial G_{0} x . y \notin G_{0} x$. Then $g_{n}^{-1}(y) \rightarrow x$ by Lemma 3.1.6 and $g_{n}^{-1}(y) \notin G_{0} x$. But $g_{n}^{-1}(y) \in \partial A_{\delta}$. This is impossible by Corollary 3.2.5.

Corollary 3.2.7. If $x \in \partial \dot{A_{\delta}}$ is arcwise accessible from $A_{\delta}$, then $G_{0} x$ is either a fixed point of $G_{0}$, a Jordan curve, or it is homeomorphic with the real line $R$ (then it is unbounded). 
Proof. If $\operatorname{dim} G_{0} x=0$ then $G_{0} x=\{x\}$ (a connected 0-dimensional space is a single point) and $x$ is a fixed point of $G_{0}$. Otherwise $\operatorname{dim} G_{0} x=1$. If $x$ is arcwise accessible from $A_{\delta}, G_{0} x$ is an 1-manifold by Proposition 3.2.4. Besides, $G_{0} x$ is closed by Corollary 3.2.6. $G_{0} x$ is connected since $G_{0}$ is connected. So $G_{0} x$ is a Jordan curve when it is bounded and it is homeomorphic with the real line $R$ when it is unbounded. (When it is bounded, it is compact. Otherwise it is a non-compact component (see Proposition 1.2.10).

Proposition 3.2.8. For every $\delta>0$, let $A_{\delta}$ denote an arbitrary component of $\bar{M}_{\delta}^{c}$ in $R^{2}$. Then $\partial A_{\delta}$ either consists only of fixed points of $G_{0}$, or it is a one-dimensional orbit $G_{0} x$ which is either a Jordan curve or homeomorphic with the real line $R$ (then it is unbounded). In case $\partial A_{\delta}$ only consists of fixed points of $G_{0}$, $\partial A_{\delta}=\partial B_{\delta, x_{0}}$ and $M_{\delta}=B_{\delta, x_{0}}$.

Proof. If there is a point $x \in \partial A_{\delta}$ which is not a fixed point of of $G_{0}$, then $G_{0} x$ is one-dimensional. When $x^{\prime}$ is sufficiently closed to $x, G_{0} x^{\prime}$ is also one-dimensional. Without loosing the generality, we can choose $x$ such that $x \in \partial A_{\delta}$ and $x$ is arcwise accessible from $A_{\delta}$ since there is a dense set of $\partial A_{\delta}$ which is arcwise accessible from $A_{\delta}$ (see Corollary 1.2.7).

Then $G_{0} x$ is either a Jordan curve or it is homeomorphic with $R$ (then it is unbounded). We need only to show that $\partial A_{\delta}=G_{0} x$.

It is easy to prove that $G_{0} x$ separates $R^{2}$ if we consider the $G_{0} x$ on the sphere $S^{2}$. By this fact, it is easy to prove that $\partial A_{\delta}=G_{0} x$.

In case $\partial A_{\delta}$ consists only of fixed points of $G_{0}$, every point of $\partial A_{\delta}$ is a point of $\partial B_{\delta, x_{0}}$ by Proposition 3.1.5. But $\partial A_{\delta}$ must be whole $\partial B_{\delta, x_{0}}$ by the fact that $\partial A_{\delta}$ separate $M_{\delta}$ and $A_{\delta}$ in $R^{2}$. Then it is trivial to show that $M_{\delta}=B_{\delta, x_{0}}$.

Proposition 3.2.9. For every $\delta>0$, let $A_{\delta}$ denote an arbitrary component of $\bar{M}_{\delta}^{c}$. Then for every $\gamma \in(0, \delta)$, there is a unique component $A_{\gamma}$ of $\bar{M}_{\gamma}^{c}$ such that $\bar{A}_{\delta}$ is a subset of $A_{\gamma}$. For every point $x \in \partial A_{\delta}$, there exists a point $x_{\gamma} \in \partial A_{\gamma}$ such that $x_{\gamma} \rightarrow x$ as $\gamma \rightarrow \delta$.

Proof. It is trivial to show that for every $\gamma \in(0, \delta)$, there exists a unique component $A_{\gamma}$ of $\bar{M}_{\gamma}^{c}$ such that $A_{\delta}$ is a subset of $A_{\gamma}$. This gives that $\bar{A}_{\delta}$ is a subset of $\bar{A}_{\gamma}$. But $\partial A_{\delta}$ does not intersect $\partial A_{\gamma}$ by Proposition 3.1.3. So $\bar{A}_{\delta}$ is a subset of $A_{\gamma}$.

For any point $x \in \partial A_{\delta}$, let $d_{\gamma}$ denote the distance between $x$ and $\partial A_{\gamma}$. Then $d_{\gamma}>0$ for every $\gamma \in(0, \delta)$ and $d_{\gamma}$ is a decreasing function of $\gamma$ when $\gamma$ is increasing to $\delta$. So $d=\lim _{\gamma \uparrow \delta} d_{\gamma}$ exists. Let us show that $d=0$.

If this is not true, then $d>0$. Then there is a neighborhood of $x$ such that every point of the neighborhood is in the $A_{\gamma}$, for all $\gamma \in(0, \delta)$. This is to say that there is a neighborhood of $x$ such that every point of the neighborhood is in $\bar{M}_{\gamma}^{c}$ for all $\gamma \in(0, \delta)$. This contradicts with the fact that $x \in \partial M_{\delta}$ (Note that $M_{\delta}=\bigcup_{\gamma<\delta} M_{\gamma}$ ). 
So $d=0$. This gives that for every $\gamma \in(0, \delta)$, there exists a point $x_{\gamma}$ in the $\partial A_{\gamma}$ such that $x_{\gamma} \rightarrow x$ as $\gamma \rightarrow \delta$. Proposition 3.2.9 is proved.

LEMMA 3.2.10. Let $x$ and $y$ be two points of $R^{2}$. If there are two sequences $x_{n}$ and $y_{n}$ such that (1): $G_{0} x_{n}=G_{0} y_{n}$, for all $n ;(2): x_{n} \rightarrow x$ and $y_{n} \rightarrow y$, when $n \rightarrow \infty$, then $x \in \overline{G_{0} y}$ and $y \in \overline{G_{0} x}$.

Proof. Let $U, V$ be two open neighborhoods of $e$ in $G$ such that $g^{-1} V g \subset U$ for all $g \in G$ by the uniformity of the group $G$. Then $U y$ is an open neighborhood of $y$ since $G$ acts openly on $R^{2}$. This gives that $y_{n} \in U y$ when $n$ is sufficiently large. For same reason, we have $x_{n} \in V x$ when $n$ is sufficiently large.

Since $x_{n}$ and $y_{n}$ are in same orbit we have $x_{n}=g_{n}\left(y_{n}\right)$ for some $g_{n} \in G_{0}$.

This gives that $x_{n}=g_{n}\left(y_{n}\right) \in V x$ when $n$ is sufficiently large. Then $y_{n} \in g_{n}^{-1} V x=$ $g_{n}^{-1} V g_{n} g_{n}^{-1}(x) \subset U g_{n}^{-1}(x)$. Then $g_{n}^{-1}(x) \in U^{-1} y_{n} \subset U^{-1} U y$ when $n$ is sufficiently large. But $U^{-1} U y$ can be taken to be an arbitrarily small open neighborhood of $y$ by choosing $U$ sufficiently small. Therefore we have shown that $g_{n}^{-1}(x) \rightarrow y$ where $g_{n}^{-1} \in G_{0}$. This gives that $y \in \overline{G_{0} x}$. By Lemma 3.1.6, we have also $x \in \overline{G_{0} y}$.

Proposition 3.2.11. For $\delta>0$, if $\bar{M}_{\delta}^{c}$ has two different components $A_{\delta}$ and $A_{\delta}^{\prime}$ in $R^{2}$, then for every $\gamma \in(0, \delta)$, there are two different components $A_{\gamma}$ and $A_{\gamma}^{\prime}$ of $\bar{M}_{\gamma}^{\mathrm{c}}$, such that $\bar{A}_{\delta} \subset A_{\gamma}$ and $\bar{A}_{\delta}^{\prime} \subset A_{\gamma}^{\prime}$.

Proof. By Proposition 3.2.9, for every $\gamma \in(0, \delta)$, there is a unique component $A_{\gamma}$ of $\bar{M}_{\gamma}^{c}$ such that $\bar{A}_{\delta} \subset A_{\gamma}$ and a unique component $A_{\gamma}^{\prime}$ of $\bar{M}_{\gamma}^{c}$ such that $\bar{A}_{\delta}^{\prime} \subset A_{\gamma}^{\prime}$.

If $A_{\gamma}$ and $A_{\gamma}^{\prime}$ are two different components of $\bar{M}_{\gamma}^{c}$ for all $\gamma \in(0, \delta)$, then the proposition is proved.

If $A_{\gamma}=A_{\gamma}^{\prime}$, for some $\gamma \in(0, \delta)$, let $\gamma_{0}=\sup \left\{\gamma \in(0, \delta)\right.$, with $\left.A_{\gamma}=A_{\gamma}^{\prime}\right\}$. Then $\gamma_{0} \leqq \delta$.

We show that $A_{\gamma_{0}}=A_{\gamma_{0}}^{\prime}$.

If this is not true, then $A_{\gamma_{0}}$ and $A_{\gamma_{0}}^{\prime}$ are two different components of $\bar{M}_{\gamma_{0}}^{c}$ in $R^{2}$. By assumption on $\gamma_{0}$, there is a sequence $\gamma_{n} \uparrow \gamma_{0}$ such that $\bar{A}_{\gamma_{0}}$ and $\bar{A}_{\gamma_{0}}^{\prime}$ are in one component $A_{\gamma_{n}}\left(=A_{\gamma_{n}}^{\prime}\right)$ of $\bar{M}_{\gamma_{n}}^{\text {c }}$ in $R^{2}$ for all $n$.

It is easy to see that none of $\partial A_{\gamma_{0}}$ and $\partial A_{\gamma_{0}}^{\prime}$ only consists of fixed points of $G_{0}$ (see Proposition 3.2.8). So $\partial A_{\gamma_{0}}=G_{0} x$ and $\partial A_{\gamma_{0}}^{\prime}=G_{0} y$, with $x \in \partial A_{\gamma_{0}}$ and $y \in \partial A_{\gamma_{0}}^{\prime}$, and each of them is either a Jordan curve or homeomorphic with the real line. When $\gamma_{n} \uparrow \gamma_{0}$, there are two sequences $x_{n}, y_{n} \in \partial A_{\gamma_{n}}$ such that $x_{n} \rightarrow x$ and $y_{n} \rightarrow y$ by Proposition 3.2.9. Then neither $\partial A_{\gamma_{n}}$ consists only of fixed points of $G_{0}$ and $\partial A_{\gamma_{n}}=G_{0} x_{n}=G_{0} y_{n}$ is a one-dimensional orbit which is either a Jordan curve or is homeomorphic with the real line, when $n$ is sufficiently large.

By Lemma 3.2.10, we have $y \in \overline{G_{0} x}=\partial A_{\gamma_{0}}$ and $x \in \overline{G_{0} y}=\partial A_{\gamma_{0}}^{\prime}$. Then $\partial A_{\gamma_{0}}=\partial A_{\gamma_{0}}^{\prime}$ and this is a contradiction.

This gives that $\gamma_{0}<\delta$. 
For every $\gamma \in\left(\gamma_{0}, \delta\right)$, there are two components $A_{\gamma}$ and $A_{\gamma}^{\prime}$ such that $\bar{A}_{\delta} \subset A_{\gamma}$ and $\bar{A}_{\delta}^{\prime} \subset A_{\gamma}^{\prime}$. Choose a point $a \in A_{\delta}$ and a point $b \in A_{\delta}^{\prime}$. Draw an arc from $a$ to $b$ in $A_{\gamma_{0}}\left(=A_{\gamma_{0}}^{\prime}\right)$ which is a domain. Then the arc intersects a point $x_{\gamma} \in \partial \bar{M}_{\gamma}^{c}=\partial \bar{M}_{\gamma}$ for every $\gamma \in\left(\gamma_{0}, \delta\right)$. Choose a sequence $\gamma_{n} \downarrow \gamma_{0}\left(\gamma_{n} \in\left(\gamma_{0}, \delta\right)\right)$. Then at least a subsequence of $x_{\gamma_{n}}$ converges to a point $x$ on the arc. Without loosing the generality, assume that $x_{\gamma_{n}} \rightarrow x$. Since $x_{\gamma_{n}} \in \partial \bar{M}_{\gamma_{n}}$ for every $n$ and $\bar{M}_{\gamma_{m}} \subset M_{\gamma_{n}} \subset \bar{M}_{\gamma_{n}}$ for $m>n$, it is easy to see that $x \in M_{\gamma_{n}}-M_{\gamma_{0}}$ for every $n$.

On the other hand, $x$ is a point of $\partial M_{\alpha}$ for some $\alpha$ (Proposition 3.1.5), so $\gamma_{0} \leqq \alpha<\gamma_{n}$. Let $n \rightarrow \infty$, then $\alpha=\gamma_{0}$. This is to say, the arc from $a$ to $b$ intersects a point of $\partial M_{\gamma_{0}}$. This is a contradiction since we have chosen the arc to be totally in $\bar{M}_{\gamma_{0}}^{c}$. Proposition 3.2.11 is proved.

LEMMA 3.2.12. If an orbit $G_{0} x$ is compact, then for every neighborhood $\mathscr{W}$ of $G_{0} x$, there exists a neighborhood of $x$, such that, if $y$ is in this neighborhood of $x$, then $G_{0} y \subset \mathscr{W}$.

Proof. Let $\mathscr{W}$ be a neighborhood $G_{0} x$. Then by Proposition 1.2.2, there exists an open neighborhood $U$ of $e$ of $G$, such that $U G_{0} x$ is in $\mathscr{W}$. Since $G$ is a uniform group, there is another open neighborhood $V$ of $e$ of $G$, such that $g^{-1} V g \subset U$, $\forall g \in G_{0}$. $G$ acts openly on $R^{2}$, so $V x$ is an open neighborhood of $x$ in $R^{2}$. If $y \in V x$, then for every $g \in G_{0}, g(y) \in g V x=g V g^{-1} g(x) \subset U g(x) \subset U G_{0} x \subset \mathscr{W}$. This is to say that $G_{0} y$ is in $\mathscr{W}$.

REMARK. If a Jordan curve $C$ separates $R^{2}$ into two connected and open sets, one bounded and the other unbounded and a point $x$ is in the bounded set, we say that the Jordan curve $C$ surrounds the point $x$. If a set $B$ is in the bounded set, we say that the Jordan curve $C$ surrounds the set $B$,

PROPOSITION 3.2.13. If $M_{\delta}$ is a bounded set in $R^{2}$ and $A_{\delta}$ is an arbitrary component of $\bar{M}_{\delta}^{c}$, then $\partial A_{\delta}$ either only consists of fixed points of $G_{0}$ or it is an orbit $G_{0} x$ which is a Jordan curve and which surrounds the interior of the set $\bar{M}_{\delta}$.

PROOF. When $M_{\delta}$ is a bounded set in $R^{2}$ and $A_{\delta}$ is a component of $\bar{M}_{\delta}^{c}, \partial A_{\delta}$ is also bounded. If $\partial A_{\delta}$ does not consist of fixed points of $G_{0}$, then it is an orbit $G_{0} x$ which is a Jordan curve by Proposition 3.2.8. Suppose that the Jordan curve $\partial A_{\delta}=G_{0} x$ separates $R^{2}$ into two connected and open sets $\mathscr{D}_{1}$ and $\mathscr{D}_{2}$ and let $\mathscr{D}_{1}$ denote the bounded set. We show that $x_{0} \in \mathscr{D}_{1}$.

If this is not true, then $x_{0} \in \mathscr{D}_{2}$. Then it is easy to see that both $B_{x_{0}, \delta}$ and $M_{\delta}$ are in $\mathscr{D}_{2}$ and $\mathscr{D}_{1}=A_{\delta}$.

For every $\gamma \in(0, \delta)$, we know by Proposition 3.2.9 that there exists a unique component $A_{\gamma}$ of $\bar{M}_{\gamma}^{c}$ such that $\bar{A}_{\delta}$ is a subset of $A_{\gamma}$.

In fact, for these sets $A_{\gamma}, \gamma \in(0, \delta)$, we have that $\bar{A}_{\gamma^{\prime}} \subset A_{\gamma}$ if $0<\gamma<\gamma^{\prime}<\delta$.

When $\gamma$ is sufficiently close to $\delta$, there exists a point $x_{\gamma} \in \partial A_{\gamma}$ such that $x_{\gamma}$ is close 
to $x$. Then $G_{0} x_{\gamma}$ is one-dimensional and close to the Jordan curve $\partial A_{\delta}=G_{0} x$ (see Lemma 3.2.12).

Then $G_{0} x_{\gamma}=\partial A_{\gamma}$ is also a Jordan curve and it surrounds the Jordan curve $\partial A_{\delta}=G_{0} x$. For every $\gamma^{\prime} \in(\gamma, \delta)$, we have that the Jordan curve $\partial A_{\gamma}=G_{0} x_{\gamma}$ surrounds the Jordan curve $\partial A_{\gamma^{\prime}}=G_{0} x_{\gamma^{\prime}}$ which surrounds the Jordan curve $\partial A_{\delta}=G_{0} x$.

If for all $\gamma \in(0, \delta), \partial A_{\gamma}$ is an orbit $G_{0} x_{\gamma}$ which is a Jordan curve and surrounds the Jordan curve $\partial A_{\delta}=G_{0} x$, then when $\gamma \rightarrow 0, G_{0} x_{\gamma}=\partial A_{\gamma}$ cannot be close to the fixed point $x_{0}$ of $G_{0}$, which is obviously a contradiction.

So there exists an $\gamma \in(0, \delta)$, such that $\partial A_{\gamma}$ either only consists of fixed points of $G_{0}$ or $\partial A_{\gamma}$ is an orbit $G_{0} x_{\gamma}$, which is a Jordan curve but does not surround the Jordan curve $\partial A_{\delta}$. Let $\gamma_{0}$ be the supremum of all those $\gamma$.

If $\partial A_{\gamma_{0}}$ only consists of fixed points of $G_{0}$, then $\partial A_{\gamma_{0}}=\partial B_{\gamma_{0}, x_{0}}$. Consider an $\gamma \in\left(\gamma_{0}, \delta\right)$, which is sufficiently close to $\gamma_{0}$. Then $\partial A_{\gamma}$ must be close to a point of $\partial B_{\gamma_{0}, x_{0}}$. This contradicts with the fact that $\partial A_{\gamma}$ surrounds $\partial A_{\delta}$.

If $\partial A_{\gamma_{0}}$ is an orbit $G_{0} x_{\gamma_{0}}$ which is a Jordan curve but does not surround the Jordan curve $\partial A_{\delta}$, then $\partial A_{\gamma_{0}}$ must surround the point $x_{0}$, since if not, the bounded open connected part of the plane separated by $\partial A_{\gamma_{0}}$ must be $A_{\gamma_{0}}$. But $A_{\gamma_{0}}$ contains $A_{\delta}$, this implies that $\partial A_{\gamma_{0}}$ surrounds $\partial A_{\delta}$.

Consider an $\gamma \in\left(\gamma_{0}, \delta\right)$ and $\gamma$ is sufficiently close to $\gamma_{0}$. Then $\partial A_{\gamma}$ must be close to $\partial A_{\gamma_{0}}$ which contradicts the fact that $\partial A_{\gamma}$ surrounds $\partial A_{\delta}$ as well.

So $\partial A_{\gamma_{0}}$ is an orbit $G_{0} x_{\gamma_{0}}$ which is a Jordan curve and which surrounds the $\partial A_{\delta}=G_{0} x$. Then for every $\gamma \in\left(0, \gamma_{0}\right)$ which is sufficiently close to $\gamma_{0}, \partial A_{\gamma}$ also surrounds $\partial A_{\delta}$. This contradicts with the assumption on $\gamma_{0}$.

These give that $\partial A_{\delta}$ surrounds $x_{0}$. Since $\partial A_{\delta}$ separates the interior of $\bar{M}_{\delta}$ and $A_{\delta}$, it is easy to see that $\partial A_{\delta}$ surrounds the interior of $\bar{M}_{\delta}$. The proposition is proved.

\subsection{THE SETS $\partial M_{\delta}$ AND $\partial \bar{M}_{\delta}$.}

Proposition 3.3.1. For every $\delta>0$ with $\partial \bar{M}_{\delta} \neq \emptyset$ and for every $x \in \partial \bar{M}_{\delta}$, the orbit $G_{0} x$ is either a fixed points of $G_{0}$, a Jordan curve, or it is homeomorphic with the real line $R$ (then it is unbounded).

Proof. By Proposition 3.2.8 and the fact that $\bar{M}_{\delta}^{c}=\cup A_{\delta}$, every $x \in \partial \bar{M}_{\delta}$ is a point of $\partial A_{\delta}$ for some $A_{\delta}$.

Proposition 3.3.2. The set $D=\left\{x \in R^{2}: \exists \delta>0\right.$ with $\left.x \in \partial \bar{M}_{\delta}\right\}=\cup \partial \bar{M}_{\delta}$ is dense in $R^{2}$.

Proof. For every $x$ of $R^{2}$, there exists a unique $\delta>0$ such that $x \in \partial M_{\delta}$. By the proof of the Proposition 3.1.5, we know that $\delta=\sup \left\{\gamma, x \notin M_{\gamma}\right\}$ and for every $\gamma \in(0, \delta)$, we have that $x \notin \bar{M}_{\gamma}$. 
For each $\gamma \in(0, \delta)$, let $A_{\gamma}$ be the unique component of $\bar{M}_{\gamma}^{c}$ in $R^{2}$ such that $x \in A_{\gamma}$. Then for these sets $A_{\gamma}$, we even have $x \in A_{\gamma^{\prime}} \subset \bar{A}_{\gamma^{\prime}} \subset A_{\gamma}$ for any two $\gamma$ and $\gamma^{\prime}$ with $0<\gamma<\gamma^{\prime}<\delta$.

Let $d_{\gamma}$ denote the distance between the point $x$ and the $\partial A_{\gamma}$. Then $d_{\gamma}>0$ and $d_{\gamma}$ is a decreasing function of $\gamma$ when $\gamma$ is increasing to $\delta$. So $d=\lim _{\gamma \uparrow \delta} d_{\gamma}$ exists and in same way as in the proof of Proposition 3.2.9, we can show that $d=0$. This gives that there is a sequence $\gamma_{n} \uparrow \delta$ and a sequence $x_{n} \in \partial A_{\gamma_{n}}$, such that $x_{n} \rightarrow x$. The proposition is proved.

Proposition 3.2.13 gives us the following.

Proposition 3.3.3. If $M_{\delta}$ is bounded in $R^{2}$, then $\partial M_{\delta}$ is a Jordan curve. This Jordan curve separates $R^{2}$ into two connected and open sets, one bounded and the other unbounded, $M_{\delta}$ is the bounded set.

Proof. Proposition 3.2.13 gives us that if $M_{\delta}$ is bounded and $A_{\delta}$ is a component of the $\bar{M}_{\delta}^{\mathrm{c}}$, then $\partial A_{\delta}$ either only consists of fixed points of $G_{0}$ or it is an orbit $G_{0} x$ which is a Jordan curve surrounding the interior of the set $\bar{M}_{\delta}$.

In case there is a component $A_{\delta}$ of the $\bar{M}_{\delta}^{c}$, whose boundary only consists of fixed points of $G_{0}$, we have $\partial A_{\delta}=\partial B_{\delta, x_{0}}$ and $M_{\delta}=B_{\delta, x_{0}}$ (Proposition 3.2.8), and the proposition is obviously true.

So we need only to consider the case when $\partial A_{\delta}$ is an orbit $G_{0} x$ which is a Jordan curve surrounding the interior of the set $\bar{M}_{\delta}$, for every component $A_{\delta}$ of the $\bar{M}_{\delta}^{c}$.

Notice that every $\partial A_{\delta}$ has at least one common point with $\partial B_{\delta, x_{0}}$ by Proposition 3.1.5.

Then we see that there cannot exist two different components $A_{\delta}$ and $A_{\delta}^{\prime}$ of $\bar{M}_{\delta}^{c}$ in $R^{2}$. Otherwise, $\partial A_{\delta}$ and $\partial A_{\delta}^{\prime}$ intersect each other.

In other words, we have shown that $\bar{M}_{\delta}^{c}$ is an open and connected set and $\partial\left(\bar{M}_{\delta}^{c}\right)=\partial \bar{M}_{\delta}$ is a Jordan curve which separates $R^{2}$ into two connected and open sets, denoted by $\mathscr{D}_{1}$ and $\mathscr{D}_{2}$. Let $\mathscr{D}_{1}$ denote the bounded set, then $M_{\delta}$ is in $\mathscr{D}_{1}$ and $\bar{M}_{\delta}^{c}$ is equal to $\mathscr{D}_{2}$. We need only to prove that $\mathscr{D}_{1}$ is $M_{\delta}$.

If there is a point $z$ in $\mathscr{D}_{1}$, which is not in $M_{\delta}$, then $z \in \partial M_{\delta}$.

If we consider $\gamma \uparrow \delta$, then $z \in \bar{M}_{\gamma}^{c}$ for every $\gamma$. But on the other hand, $\partial \vec{M}_{\gamma}$ is a Jordan curve, surrounding the interior of the $\bar{M}_{\gamma}$, and which can be chosen arbitrarily close to the Jordan curve $\partial \bar{M}_{\delta}$ (see the proof of the Proposition 3.2.2). This gives that that $z \in \bar{M}_{\gamma}^{0}$ when $\gamma$ is sufficiently close to $\delta$. This is a contradiction. Proposition 3.3.3 is proved.

LEMMA 3.3.4. If there exists $\delta>0$, such that $\bar{M}_{\delta}^{c}=\emptyset$, then there exists a smallest value $\delta_{0}$ of all those $\delta>0$ such that $\bar{M}_{\delta}^{c}=\emptyset$. For every $\delta>\delta_{0}$, we have $M_{\delta}=R^{2}$.

Proof. If there exists an $\delta>0$ such that $\bar{M}_{\delta}^{c}=\emptyset$, let $\delta_{0}=\inf \left\{\delta, \bar{M}_{\delta}^{c}=\emptyset\right\}$. We 
show that $\bar{M}_{\delta_{0}}^{c}=\emptyset$. If this is not true, there is a point $x \in \bar{M}_{\delta_{0}}^{c}$. For every $\delta>\delta_{0}$, we have $x \in M_{\delta}$ (in fact, by the assumption on $\delta_{0}$, it is easy to see that for every $\delta>\delta_{0}$ we have $M_{\delta}=R^{2}$ ). Choose a sequence $\delta_{n} \downarrow \delta_{0}$. Then there exists a sequence $g_{n} \in G_{0}$ such that $g_{n}(x) \in B_{\delta_{n}, x_{0}}$. But on the other hand, notice that $g_{n}(x) \notin \bar{B}_{\delta_{0}, x_{0}}$. So there is a subsequence of $g_{n}(x)$, which converges to a point $y$ of $\bar{B}_{\delta_{0}, x_{0}}$. Without loosing the generality, assume that $g_{n}(x) \rightarrow y \in \bar{B}_{\delta_{0}, x_{0}}$.

Since $y \in \bar{M}_{\delta_{0}}$ and $g_{n}^{-1}(y) \rightarrow x$ we get that $x \in \bar{M}_{\delta_{0}}$. This is a contradiction. We have proved that $\bar{M}_{\delta_{0}}^{c}=\emptyset$. Then $\delta_{0}$ is the smallest value of all $\delta>0$ such that $\bar{M}_{\delta}^{0}=\emptyset$. For every $\delta>\delta_{0}$, we have $M_{\delta}=R^{2}$ by Proposition 3.1.3.

Proposition 3.3.5. For $\delta>0$ such that $\bar{M}_{\delta}^{c} \neq \emptyset, \bar{M}_{\delta}^{c}$ is open and connected.

Proof. By Lemma 3.3.4, if there exists an $\delta>0$, such that $\bar{M}_{\delta}^{c}=\emptyset$, then there exists a smallest value $\delta_{0}$ of all $\delta>0$ such that $\bar{M}_{\delta}^{c}=\emptyset$. For every $\delta>\delta_{0}$, we have $\bar{M}_{\delta}^{c}=\emptyset$.

We need only to show that $\bar{M}_{\delta}^{c}$ is open and connected for all $\delta \in\left(0, \delta_{0}\right)$ where $0<\delta_{0} \leqq \infty$.

If there is an $\delta \in\left(0, \delta_{0}\right)$ such that $\bar{M}_{\delta}^{c}$ has two different components $A_{\delta}$ and $A_{\delta}^{\prime}$ in $R^{2}$, then by Proposition 3.2.11, for every $\gamma \in(0, \delta)$, there are two different components $A_{\gamma}$ and $A_{\gamma}^{\prime}$ of $\bar{M}_{\gamma}^{c}$ such that $\bar{A}_{\delta} \subset A_{\gamma}$ and $\bar{A}_{\delta}^{\prime} \subset A_{\gamma}^{\prime}$.

But by Proposition 3.3.3, when $\gamma>0$ is so small such that $M_{\gamma}$ is bounded, there is only one component of $\bar{M}_{\gamma}^{c}$. This is a contradiction. Proposition 3.3.5 is proved.

COROLlaRY 3.3.6. For $\delta>0$ such that $\bar{M}_{\delta}^{c} \neq \emptyset, \partial \bar{M}_{\delta}$ either only consists of fixed points of $G_{0}$ or it is a one-dimensional orbit $G_{0} x$ which is either a Jordan curve or homeomorphic with the real line (then it is unbounded).

Proof. See Proposition 3.2.8 and Proposition 3.3.5.

Proposition 3.3.7. For $\delta>0$ such that $\bar{M}_{\delta}^{c} \neq \emptyset$, we have $\partial M_{\delta}=\partial \bar{M}_{\delta}$.

Proof. By Lemma 3.3.4, let $\delta_{0}$ be the smallest value of all $\delta>0$ such that $\bar{M}_{\delta}^{c}=\emptyset$. Then for every $\delta>\delta_{0}$, we have $\partial M_{\delta}=\partial \bar{M}_{\delta}=\emptyset$. We need only to show that $\partial M_{\delta}=\partial \bar{M}_{\delta}$, for all $\delta \in\left(0, \delta_{0}\right)$, where $0<\delta_{0} \leqq \infty$.

By Proposition 3.3.3, this is true when $\delta>0$ is so small such that $M_{\delta}$ is bounded.

If the statement is not true for some $\delta \in\left(0, \delta_{0}\right)$, then there exists a point $x \in \partial M_{\delta}$ such that $x \notin \partial \bar{M}_{\delta} \neq \emptyset$. Then $\partial \bar{M}_{\delta}$, which obviously does not consist of fixed points of $G_{0}$, is an orbit $G_{0} y$ of a point $y \in \partial \bar{M}_{\delta}$, which is either a Jordan curve or is homeomorphic with the real line.

Choose a sequence $\gamma_{n} \uparrow \delta$. Then there are two sequences $x_{n}, y_{n} \in \partial \bar{M}_{\gamma_{n}}$ such that $x_{n} \rightarrow x, y_{n} \rightarrow y$ (see the proof of Proposition 3.3.2). 
Since $y$ is not a fixed point of $G_{0}$, we see that when $n$ is sufficiently large, $y_{n}$ is not a fixed point of $G_{0}$. This gives that $\partial \bar{M}_{\gamma_{n}}=G_{0} y_{n}=G_{0} x_{n}$ (see Corollary 3.3.6).

Then by Lemma 3.2.10, we get the contradiction that $x \in \partial \bar{M}_{\delta}$.

We have shown that $\partial M_{\delta}=\partial \bar{M}_{\delta}$ for all $\delta \in\left(0, \delta_{0}\right)$. The proposition is proved.

COROLLARY 3.3.8. There exists at most one $\delta_{0}>0$, such that $\partial M_{\delta_{0}} \neq \partial \bar{M}_{\delta_{0}}$ and for this $\delta_{0}$, we have $\partial \bar{M}_{\delta_{0}}=\emptyset$. Moreover, for every $\delta>\delta_{0}$, we have $\partial \bar{M}_{\delta}=\partial M_{\delta}=\emptyset$ and for every point $x$ of $R^{2}$ other than $x_{0}$, there is a unique $\delta \in\left(0, \delta_{0}\right]$, such that $x \in \partial M_{\delta}$.

\subsection{THE MAIN RESUlTS OF THIS PAPER.}

In this section, we give the proofs for our main results in this paper. Recall the general assumption on $G$ and $G_{0}$ stated at the beginning of this chapter.

LEMMA 3.4.1. There is an open, connected and dense subset $O$ of $R^{2}$, such that, for every point $x$ of $O$, the orbit $G_{0} x$ is either a fixed point of $G_{0}$, a Jordan curve, or it is homeomorphic with the real line $R$ (then it is unbounded).

Proof. Corollary 3.3.8 tells us that $\partial M_{\delta}=\partial \bar{M}_{\delta}$ holds for all $\delta>0$ except at most one $\delta_{0}$.

Consider this eventual $\delta_{0}$. Then $\partial \bar{M}_{\delta_{0}}=\emptyset$. That is to say that $\bar{M}_{\delta_{0}}=R^{2}$. Let $O=M_{\delta_{0}}=R^{2}-\partial M_{\delta_{0}}$. Then $O$ is an open, connected $\left(M_{\delta_{0}}\right.$ is open and connected by definition) and dense subset of $R^{2}$.

For every $x \in O, x \neq x_{0}$, there is an $\delta \in\left(0, \delta_{0}\right)$ such that $x \in \partial M_{\delta} . \bar{M}_{\delta}^{c}$ is not the empty set, so $x \in \partial M_{\delta}=\partial \bar{M}_{\delta}$. Then the orbit $G_{0} x$ is either a fixed point of $G_{0}$, a Jordan curve, or it is homeomorphic with the real line $R$ (then it is unbounded) (see Proposition 3.3.1).

LEMMA 3.4.2. For $\delta>0$ such that $\bar{M}_{\delta}^{c} \neq \emptyset$, no point $x \in \partial M_{\delta}$ is a fixed point of $G_{0}$ unless $G_{0}$ fixes every point of $R^{2}$.

ProOF. If $\bar{M}_{\delta}^{c} \neq \emptyset$ and there is an $x \in \partial M_{\delta}$ which is a fixed point of $G_{0}$, then $\partial M_{\delta}=\partial \bar{M}_{\delta}=\partial B_{\delta, x_{0}}$ only consists of fixed points of $G_{0}$.

Consider an arbitrary point $y \in \partial B_{\delta, x_{0}}$ and a neighborhood of $y$. If there is a point $z$ of this neighborhood which is not a fixed point of $G_{0}$, then $G_{0} z$ is a Jordan curve, surrounding both $x_{0}$ and $y$. But if we choose $z$ sufficiently close to $y$, the Jordan curve $G_{0} z$ is so small that it cannot surround $x_{0}$. This shows that a small neighborhood of $y$ consists only of fixed points of $G_{0}$, for every $y \in \partial B_{\delta, x_{0}}$. Then when $\gamma$ is sufficiently close to $\delta$, every point of $\partial B_{\gamma, x_{0}}$ is a fixed point of $G_{0}$.

In other words, the set of all $\delta>0$ such that every point of $\partial B_{\delta, x_{0}}$ is a fixed point of $G_{0}$ is an open set of the half real line $R^{+}$.

Let $\gamma_{0}=\inf \left\{\gamma, \partial B_{\gamma, x_{0}}\right.$ only consists of fixed points of $\left.G_{0}\right\}$. Then it is easy to see that every point of $\partial B_{\gamma_{0}, x_{0}}$ is a fixed point of $G_{0}$. This gives $\gamma_{0}=0$. 
Let $\gamma_{1}=\sup \left\{\gamma, \partial B_{\gamma, x_{0}}\right.$ only consists of fixed points of $\left.G_{0}\right\}$. Then it is also easy to see that every point of $\partial B_{\gamma_{1}, x_{0}}$ is a fixed point of $G_{0}$. This gives $\gamma_{1}=\infty$.

Then we have that every point of $R^{2}$ is a fixed point of $G_{0}$.

THEOREM 3.4.3. If $G_{0}$ does not fix every point of $R^{2}$, there is an open, connected and dense subset $O$ of $R^{2}$, such that, for every point $x$ of $O$, the orbit $G_{0} x$ is either a Jordan curve or homeomorphic with the real line $R$ (then it is unbounded).

ProOF. See the proof of Lemma 3.4.1 and Lemma 3.4.2.

THEOREM 3.4.4. If $G_{0}$ does not fix every point of $R^{2}$, then $G_{0}$ fixes at most two points of $R^{2}$.

Proof. Let $G_{0}$ do not fix every point of $R^{2}$. By Lemma 3.3.4 and Lemma 3.4.2, if there is another fixed point $x$ of $G_{0}$ than $x_{0}$, then $x \in \partial M_{\delta_{0}}$ where $\delta_{0}$ is the smallest value of all $\delta>0$ such that $\bar{M}_{\delta}^{c}=\emptyset$.

Then it is easy to show that $\partial M_{\delta_{0}}=\overline{G_{0} x}=\{x\}$ (notice the assumption on $\delta_{0}$ and see Lemma 3.2.10 and the proof of Proposition 3.3.2).

Any other point $y$ than $x$ and $x_{0}$ is a point of $\partial M_{\delta}$ with $0<\delta<\delta_{0}$. Then $y$ is not a fixed point of $G_{0}$ (Lemma 3.4.2).

COROLlary 3.4.5. In case there are exactly two fixed points of $G_{0}$ in $R^{2}$, for every other point $x$ of $R^{2}$ than the two fixed points, the orbit $G_{0} x$ is either a Jordan curve or homeomorphic with the real line (then it is unbounded).

THEOREM 3.4.6. If $G_{0}$ acts effectively on $R^{2}$, then $G_{0}$ is a commutative subgroup of $G$.

Proof. If $G_{0}$ acts effectively on $R^{2}$, then $G_{0}$ does not fix every point of $R^{2}$ unless $G_{0}=\{e\}$ which obviously is a commutative subgroup of $G$. So if $G_{0} \neq\{e\}$, then by Theorem 3.4.3, there is an open, connected and dense subset $O$ of $R^{2}$, such that, for every point $x$ of $O$, the orbit $G_{0} x$ is either a Jordan curve or homeomorphic with the real line. If we can show that $G_{0}$ acts commutatively on this dense set $O$ of $R^{2}$, then it is easy to see that $G_{0}$ acts commutatively on $R^{2}$ and then, since $G_{0}$ acts effectively on $R^{2}$, it is easy to show that $G_{0}$ is a commutative subgroup of G.

For every $x \in O, G_{0}$ is a connected and uniform transformation group on $G_{0} x$ which is homeomorphic with $C$ or $R$. By the results from Chapter $2, G_{0}$ is a commutative group if $G_{0}$ acts effectively on $G_{0} x$.

If $G_{0}$ does not act effectively on $G_{0} x$ (as a transformation group), let $H$ be the subgroup of $G_{0}$, which consists of those transformations of $G_{0}$, which leave $G_{0} x$ fixed. Then $H$ pointwise is a closed normal subgroup of $G_{0}$ on the orbit and we see that $G_{0} / H$ is a connected uniform group when $G_{0}$ is connected and uniform (see 
[4], p. 235). The natural map of $G_{0}$ onto $G_{0} / H$ is a continuous and open homeomorphism. So $G_{0} / H$ is connected, uniform and acts effectively as a transformation group on $G_{0} x$. By the results from Chapter $2, G_{0} / H$ acts commutatively on $G_{0} x$. Then it is easy to see that $G_{0}$ acts commutatively on $G_{0} x$.

REMARK. (1) We have proved, without assuming that $G_{0}$ acts effectively on $R^{2}$, that as long as $G_{0}$ does not fix every point of $R^{2}$, then "almost" every orbit $G_{0} x$ is as good as either a Jordan curve or is homeomorphic with the real line. If we know that $G_{0}$ acts effectively on one of those good orbits, that would be enough to prove that $G_{0}$ is a commutative group, by the result from Chapter 2 .

(2) Under the same conditions on $G$ and $G_{0}$, most propositions and theorems carry over to the case of the unit sphere $S^{2}$ instead of the Euclidean 2-space $R^{2}$. The proofs are the same with minor modifications and hopefully, we can even get more complete results on $S^{2}$ such as (a) if $G_{0}$ does not leave every point of $S^{2}$ fixed, then there are exactly two fixed points of $G_{0}$ on $S^{2}$, such that for every other point $x$ than the two fixed points, the orbit $G_{0} x$ is a Jordan curve.

(3) Throughout the proofs in Chapter 3, the only assumptions we used about the larger group $G$ are (a) it acts openly on $R^{2}$, that is to say, for every point $x \in R^{2}$, every open neighborhood $U$ of the unity $e$ in $G$, acting on $x$, gives an open subset $U x$ in $R^{2}$; (b) $G$ is uniform, that is to say, for every open neighborhood $U$ of the unity $e$ in $G$, there is an open neighborhood $V$ of $e$ in $G$, such that, for every transformation $f$ in $G$, we have $f V f^{-1} \subset U$. Nowhere did we need that the product $f g$ of any two transformations $f$ and $g$ in $G$ remains in $G$. So actually, it is enough to assume that $G$ is a subset of a transformation group on $R^{2}$, which contains the unity $e$ of the group, and under the topology of the group, to assume that $G$ acts openly on $R^{2}$. Then we assume that $G$ is a uniform subset of the group in the sense that for every open neighborhood $U$ of the unity $e$ in $G$, there is an open neighborhood $V$ of $e$ in $G$, such that, for every transformation $f$ in $G$, we have $f V f^{-1} \subset U$. Under these assumptions on $G$, all the propositions and theorems in Chapter 3 still are true. It should be easier to find examples of such a set of transformations on $R^{2}$ than a full group of transformations.

Acknowledgements. The author is grateful to Professor Per Enflo for suggesting the topic and to Dr. Björn Gustafsson who has spent a lot of time to discuss the problems with the author and read many early versions of the paper. Without this help, the paper would never be finished.

\section{REFERENCES}

1. D. Montgomery and L. Zippin, Topological Transformation Groups, Interscience Publishers, New York, 1955.

2. Borel et al. Seminar on Transformation Groups, Annals of Math. Surveys, 1960. 
3. Conner and Floyd, Differential Periodic Maps, Springer-verlag 1964.

4. P. Enflo, Uniform structures and square roots in topological groups, Part I, Israel J. Math. 8(1970), 230-252.

5. J. L. Kelley, General Topology, D. Van Nostrand Company Inc., 1955.

6. W. Hurewicz and H. Wallman, Dimension Theory, Princeton University Press, 1948.

7. R. L. Wilder, Topology of Manifold, Published by the American Mathematical Society, 1949.

8. Willard, General Topology, Addison-Wesley, 1970, page 222.

9. L. V. Ahlfors and L. Sario, Riemann Surfaces, Princeton University Press, 1960, p. 15.

DEPARTMENT OF MATHEMATICS

ROYAL INSTITUTE OF TECHNOLOGY

10044 S TOCKHOLM

SWEDEN 